\title{
An Emergence of a Quantum World in a Self-Organized Vacuum-A Possible Scenario
}

\author{
Vladimir A. Manasson \\ Sierra Nevada Corporation, Irvine, California, USA \\ Email: v.a.manasson@cox.net
}

How to cite this paper: Manasson, V.A. (2017) An Emergence of a Quantum World in a Self-Organized Vacuum-A Possible Scenario. Journal of Modern Physics, 8, 1330-1381.

https://doi.org/10.4236/jmp.2017.88086

Received: April 29, 2017

Accepted: July 14, 2017

Published: July 17, 2017

Copyright $\odot 2017$ by author and Scientific Research Publishing Inc. This work is licensed under the Creative Commons Attribution International License (CC BY 4.0).

http://creativecommons.org/licenses/by/4.0/

\section{(c) (7) Open Access}

\begin{abstract}
We have explored a model of vacuum self-organization based on dissipative dynamics and recurrent self-interactions. The initial state of the vacuum is assumed as self-interacting vacuum dust. The medium is dispersive and resembles dark-energy vacuum as described by general relativity. Beside selfdiffusion, vacuum dust endowed with self-attraction, resembling Newton's gravity. We explored what would happen with this medium when the strength of self-gravitation progressively increases. We observed a cascade of phase transitions. First transition occurs when self-attraction reaches the point when it can balance self-diffusion. A vortex-cellular structure emerges. Vortexes operate as self-sustained oscillators and tend to synchronize their dynamics. They form a synchronized network that possesses a universal time scale and, after zooming out, its structure acquires a form of fiber-bundle structure of electromagnetic field. With increasing self-gravitation strength, the system experiences another phase transition. The fiber-bundle structure becomes resembling that of weak nuclear field. Vacuum cells acquire spinorial dynamics. Electric charges emerge. When synchronized, the weakly interacting cells create lepton-like molecules. Oscillating charges in spinorial cells give a birth to current loops, which magnetic moment linked to the particle spin. During the next phase transition, the cell dynamics experiences another topological transformation, which is accompanied by creation of three color charges. The acquired fiber-bundle structure form resembles that of strong nuclear field. Synchronized strongly interacting vacuum cells create quark-like particles that carry color charges. We associate their complex synchronization patterns with particle flavors. We also explored statistical distributions of vacuum cells as functions of self-gravitation strength. We found that the distribution spectrum is essentially discrete, and the vacuum cells group around the states that we call super-attractive. Discrete cell distribution implies charge quantization. Synchronization transforms initial Boltzmannlike distribution into quantum-like distributions. During phase transitions, cell distributions experience transformations that can be encoded in the
\end{abstract}


chemical potentials of the corresponding states. We found that chemical potentials apparently relate to the coupling constants and mixing angles and amplitudes in the standard model.

\section{Keywords}

Elementary Particles, Standard Model, Quantized Charges, Quark Mixing, Self-Organization, Nonlinear Dynamics

\section{Introduction}

Before presenting the model, let me attract the reader's attention to a few observations made by the author and other researchers [1] [2] [3] [4].

\subsection{Is Electron a Composite Particle?}

Let us consider a thought experiment. An initially neutral vacuum volume experiences spontaneous polarization in a form of two charged clouds carrying charges $q^{+}$and $q^{-}$. Let us assume that the clouds interact with each other by pure electrostatic forces. In Figure 1, the clouds are represented by capacitor $C$. After a while the clouds recombine and dissipate (radiate) the accumulated energy in the vacuum. The latter plays the role of a matched load with impedance $\eta=\sqrt{\mu_{0} / \varepsilon_{0}} \approx 377 \Omega$, where $\mu_{0}$ is the vacuum permeability and $\varepsilon_{0}$ is the vacuum permittivity.

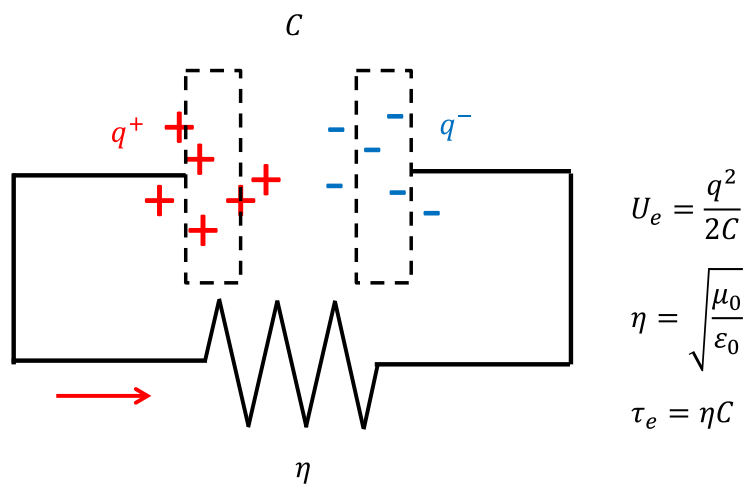

Figure 1. Cloud discharge circuit diagram. Charged clouds are represented by capacitor $C$ and the surrounding vacuum is represented by matched load $\eta$.

Electrostatic energy stored in the clouds before discharge is

$$
U_{e}=\frac{q^{2}}{2 C} \text {. }
$$

The corresponding relaxation time-constant is

$$
\tau_{e}=\eta C,
$$

and the product $U_{e} \tau_{e}$ is $C$-independent and thus is independent of the cloud geometry 


$$
U_{e} \tau_{e}=\frac{q^{2} \eta}{2}
$$

If charged clouds represent a virtual electron-positron pair, $q=e \approx 1.6 \times 10^{-19} \mathrm{C}$ (electron charge) and product (1.3) is a physical constant with dimensions of action [energy $\times$ time]

$$
\hbar_{e}=U_{e} \tau_{e}=\frac{e^{2} \eta}{2} .
$$

We use subscript $e$ to distinguish $\hbar_{e}$ from the Planck constant $\hbar$ which has the same physical dimensions.

In quantum mechanics, the pair's lifetime (dissipation time) $\tau$ depends on their energy $E$ as

$$
\hbar \approx E \tau .
$$

If the total pair's energy is due to the electrostatic forces, the two constants $\hbar$ and $\hbar_{e}$ would be of the same order $\left(\hbar_{e} \sim \hbar\right)$. However, they differ roughly by a factor of twenty! This is because the electrostatic forces contribute only a small part to the total particle energy.

More important is the fact that the ratio $\hbar / \hbar_{e}$ is a constant, and it is not only a physical constant but also a special number. With a good accuracy, it is

$$
\frac{\hbar}{\hbar_{e}} \approx 21.81 \approx \delta_{F}^{2},
$$

where $\delta_{F}=4.6692 \cdots$ is the Feigenbaum delta, a universal scaling factor describing complex transformations in dynamical systems belonging to a large universality class carrying the same name. The Feigenbaum universality was discovered decades ago [5] [6] [7] [8]. It has been observed both in theoretical models and experimentally in a number of different systems [9]-[14], but, so far, not in particle physics.

In addition to electromagnetic, electrons/positrons interact via weak nuclear forces. Their relative "strength" is provided by ratio of two physical constants $\alpha_{w} / \alpha \quad\left(\alpha_{w} \approx 1 / 29 \quad[15]\right.$ [16] is the weak coupling constant and $\alpha \approx 1 / 137 \quad$ [15] [16] is the electromagnetic coupling constant better known as the fine-structure constant). Curiously (and we believe not by accident) the ratio can also be expressed via the Feigenbaum delta

$$
\frac{\alpha_{w}}{\alpha} \approx 4.32 \approx \delta_{F} .
$$

Weak nuclear forces operate at very short distances only. It is unlikely that the two separated charged particles interact via these forces. It is more plausible to assume that the weak forces act inside the particles, i.e. that electron is a composite particle, and the weak forces keep its parts together.

\subsection{Coupling Constants as Ordered Set of Numbers}

Our encounters with the Feigenbaum constant are not completed. Another physical constant, the electroweak mixing angle $\theta_{W}$ known as the Weinberg angle can also be expressed via $\delta_{F}$. The angle is linked to the inverse $\alpha / \alpha_{w}$ ratio as 


$$
\sin ^{2} \theta_{W}=\frac{\alpha}{\alpha_{w}} \approx \delta_{F}^{-1} .
$$

The fine structure constant has been the subject of numerous speculations and its origin remains an unsolved puzzle. It is defined as [16]

$$
\alpha=\frac{e^{2}}{4 \pi \varepsilon_{0} \hbar c} .
$$

Using this definition and (1.6) one can find that [1] [3] [4]

$$
\alpha=\frac{e^{2} \eta}{4 \pi \hbar}=\frac{1}{2 \pi} \frac{\hbar_{e}}{\hbar} \approx\left(2 \pi \delta_{F}^{2}\right)^{-1} .
$$

From (1.7) and (1.9), the weak coupling constant is

$$
\alpha_{w} \approx\left(2 \pi \delta_{F}^{1}\right)^{-1}
$$

If one extends this progression toward the strong coupling constant $\alpha_{s}$, (s)he finds that

$$
\alpha_{s} \approx\left(2 \pi \delta_{F}^{0}\right)^{-1}=(2 \pi)^{-1} \approx 0.16,
$$

that is in the range of low-energy values recommend by the Particle Data Group [16].

Thus we observe that the coupling constants of strong, weak, and electromagnetic interactions can be expressed as three consecutive powers of the Feigenbaum delta (up to a multiplier $2 \pi$ ):

$$
\alpha_{j} \approx \frac{1}{2 \pi \delta_{F}^{3-j}},
$$

where $j=1,2$ and 3 respectively.

\subsection{Quark Mixing Amplitudes and Hidden Symmetry of CKM-Matrix}

Yet another example of quantum numbers that can be approximated by powers of $\delta_{F}$ is a set of the quark mixing amplitudes $V_{i j}$, where $i$ and $j$ denote different quark flavors. The $\left|V_{i j}\right|$ value set is shown below as a $3 \times 3$ CabibboKobayashi-Maskawa (CKM) matrix [17]

$$
V_{C K M}=\left[\begin{array}{lll}
\left|V_{u d}\right| & \left|V_{u s}\right| & \left|V_{u b}\right| \\
\left|V_{c d}\right| & \left|V_{c s}\right| & \left|V_{c b}\right| \\
\left|V_{t d}\right| & \left|V_{t s}\right| & \left|V_{t b}\right|
\end{array}\right] \approx\left[\begin{array}{ccc}
0.974 & 0.225 & 0.004 \\
0.225 & 0.973 & 0.041 \\
0.009 & 0.041 & 0.999
\end{array}\right]
$$

$C K M$-matrix elements can also be approximated as $\left(\delta_{F}^{-1}\right)^{p}, p=1,2$ or 3 . The approximate $V_{C K M}^{\prime}$-matrix is

$$
\begin{aligned}
V_{C K M}^{\prime} & =\left[\begin{array}{ccc}
\sqrt{1-\left(\delta_{F}\right)^{-2}-\left(\delta_{F}\right)^{-6}} & \delta_{F}^{-1} & \delta_{F}^{-3} \\
\delta_{F}^{-1} & \sqrt{1-\left(\delta_{F}\right)^{-2}-\left(\delta_{F}\right)^{-4}} & \delta_{F}^{-2} \\
\delta_{F}^{-3} & \delta_{F}^{-2} & \sqrt{1-\left(\delta_{F}\right)^{-4}-\left(\delta_{F}\right)^{-6}}
\end{array}\right](1 \\
& \approx\left[\begin{array}{lll}
0.977 & 0.214 & 0.009 \\
0.214 & 0.976 & 0.046 \\
0.009 & 0.046 & 0.999
\end{array}\right] .
\end{aligned}
$$


The expressions under the square roots have form that preserves raw/column unitarity. In future discussion, due to the smallness of $\delta_{F}^{-2}, \delta_{F}^{-4}$, and $\delta_{F}^{-6}$, we neglect their inputs in diagonal elements, and set the latter equal to 1 as shown in (1.16):

$$
V_{C K M}^{\prime}=\left[\begin{array}{ccc}
1 & \delta_{F}^{-1} & \delta_{F}^{-3} \\
\delta_{F}^{-1} & 1 & \delta_{F}^{-2} \\
\delta_{F}^{-3} & \delta_{F}^{-2} & 1
\end{array}\right] .
$$

$V_{C K M}^{\prime}$-matrix elements constitute an interesting pattern. First, the matrix possesses a diagonal symmetry. Second, each row/column comprises two out of three powers $\left(\delta_{F}^{-1}\right)^{p}, p=1,2$ or 3 , and each time the missed power is a different one. Third, $V_{\text {СКM }}^{\prime}$-matrix elements represent a subset of a $4 \times 4$ matrix where all powers are present:

$$
\left(\sum_{p=0}^{3} \delta_{F}^{-2 p}\right)^{-\frac{1}{2}}\left[\begin{array}{cccc}
1 & \delta_{F}^{-1} & \delta_{F}^{-2} & \delta_{F}^{-3} \\
\delta_{F}^{-1} & 1 & \delta_{F}^{-3} & \delta_{F}^{-2} \\
\delta_{F}^{-2} & \delta_{F}^{-3} & 1 & \delta_{F}^{-1} \\
\delta_{F}^{-3} & \delta_{F}^{-2} & \delta_{F}^{-1} & 1
\end{array}\right],
$$

In (1.17), missing elements fill the third row and third column. The term in parentheses in front of the matrix restores the raw/column unitarity.

We see that coupling constants and quark mixing amplitudes can be approximated as ordered sets of powers of the Feigenbaum delta. Could this be just a curious coincidence? We believe it is not.

\subsection{Why Self-Organization?}

The standard model does not explain why the coupling constants and mixing angles are constants and why they possess the values which we observe in experiment. Therefore, the possibility of expressing all of them through a single number, even approximately, is appealing, and more because this number is a universal constant. Why has $\delta_{F}$ not found a place in the standard model of particle interactions?

A possible explanation is that the two belong to the different frameworks. The dynamical systems with a shared feature of transition to chaotic dynamics through cascades of period-doubling bifurcations with the Feigenbaum delta $\delta_{F} \approx 4.669$ as the scaling factor belong to dissipative framework. In contrast, the standard model belongs to the Hamiltonian systems essentially based on the conservation principles.

Although, the theory predicts that cascades of period-doubling bifurcations can also occur in some nonintegrable conservative systems [12] [14] [18] [19] [20] [21] [22], this has not been confirmed in experiment. Moreover, in this case the scaling factor $\delta_{H} \approx 8.721$ essentially differs from $\delta_{F} \approx 4.669$, observed in dissipative systems.

Conservation in Hamiltonian systems is at odds with emergence. Strict time translation symmetry implies that there should neither be a big bang nor "small 
bangs/crunches" linked to the births/deaths of elementary particles. Conservation rather preserves status quo.

In contrast, if a dissipative system is far from thermodynamic equilibrium, it may exhibit an entire gamut of emergent phenomena [23] [24] [25] [26] [27]. Dissipative frameworks is more appropriate for studying emergent phenomena.

In this paper we propose a new model that describes emergence of quantum phenomena and elementary particles. We adopt a conjecture that $\delta_{F}$ is relevant to particle physics. We assume that the "primordial soup" where particles emerge and decay is a dissipative self-organized medium which we call vacuum.

One of the requirements for a dissipative system to be self-organized is that the system should be far from thermodynamic equilibrium. Numerous experiments witness that the vacuum is such a medium. It is permeated by numerous energy flows, at all levels, from quantum fluctuations to running galaxies.

Although this is not an exact parallel, Bénard flows (a network of vortices in nonequilibrium fluid) [28] may serve as a visual addition to the proposed model. This is supported by experiments where Bénard cells exhibit period-doubling bifurcations and the Feigenbaum universality [29] [30] [31] [32] [33]. Bénard cells are vortices that in essence can be described as self-sustained oscillators and tend to synchronize their dynamics, a phenomenon playing a principal role in the proposed model.

As any imitation, our model captures the most essential features only. We believe that by pursuing this approach, we may enrich our knowledge of the elementary particles and quantum fields.

\section{Cellular Dynamics}

\subsection{From Vacuum Dust to Vacuum Cells}

A simple example of spatial self-organization producing a quantized pattern is a randomly distributed iron filings placed between electromagnet poles. After field is turned on, the particles form a discrete field-line pattern. It happens under action of recurrent positive-feedback loops: small deviations from the uniform filings distribution create spots of excessive magnetic field; these spots attract more particles; the additional particles increase the local magnetic field; and so on. The filings redistribution ends when friction balances positive-feedback forces. The stronger is the external magnetic field the narrower are the field lines. The original symmetry breaks spontaneously. The locations of the field lines are arbitrary and depend on the initial filings fluctuations. Formation of the discrete field-line pattern is a phase transition. It can be seen as a "competition" among spatial positions for accumulating maximal number of particles, and the "fortunate" spots that initially have more particles are the winners. This competition resembles the Darwinian competition among the species.

More complex media can produce spatial-temporal patterns [34] [35] [36] [37] [38]. Bénard cells [28] emerging in heated liquids are among those.

In this paper, we assume that elementary particles emerge in vacuum as products of its self-organization. We assume that vacuum consists of ever mov- 
ing vacuum dust. The medium is active. The dust particles attract each other. Self-attraction is nonlinear, i.e. the denser is the dust, the stronger is self-attraction. Self-attraction competes with self-diffusion. They represent respectively a positive and negative feedbacks. Stable dynamical patterns emerge when self-attraction is balanced by self-diffusion.

The vacuum dust is in a permanent motion. The state space comprises infinitively many different modes. Luckily, the system is dissipative, which means that the modes fade out and, generally speaking, with different rates. Therefore, for many applications, it is sufficient to consider only a few principal modes with slowest dissipation rates [9] [11] [12].

We assume that most relevant modes represent vortices, local circular flows. If vortex life is long enough to perform at least several rotations, we call it a vacuum cell, or just cell. Dust migration connects vortices and forces them to synchronize in a manner of synchronization between self-sustained oscillators [39] [40] [41] [42] [43].

We also focus on intracellular radial flows. We associate them with generic charges. We assign positive sign to convergent flows, negative sign to divergent flows, and zero to pure circular flows (Figure 2). Later in the paper, depending on cell topology, we categorize generic charges as electric charges and color charges.

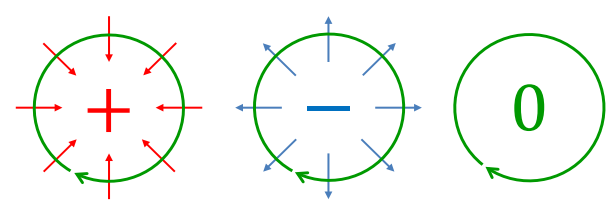

Figure 2. Charge polarity assignment (directions of radial flows are opposite to the directions of field lines adopted in electrodynamics).

We define $p$ as a probability that, in a given cell at a given time, the effective radial flow is converging. Using this convention, a cell is positively charged if $p>0.5$, it is negatively charged if $p<0.5$, and it is neutral if $p=0.5$. Positive feedback (self-attraction) increases probability $p$ with time. Negative feedback (self-diffusion) decreases probability $p$ with time.

We assume that cell evolution under combined positive- and negative-feedback can be effectively represented by a one-dimensional discrete iterated map

$$
p_{n+1}=A p_{n}\left(1-p_{n}\right) .
$$

where term $p_{n}$ represents positive feedback, term $\left(1-p_{n}\right)$ represents negative feedback, and parameter $A>1$ is a measure of the medium amplification.

Discrete points $p_{n}$ represent continuously evolving trajectories sampled stroboscopically at cross-sections with Poincaré plane (Figure 3). Fixed points $p_{\infty}$ represent single-loop (Figure 3(b)) or multi-loop (Figure 3(c)) attractors and respectively are single-valued or multiple-valued.

Dissipative evolution trajectories in the state space represent spirals (Figure 3(a)). The "stroboscopic" samplings are taken at time instants $t_{n}$, when the trajectory is crossing the selected Poincaré plane. 


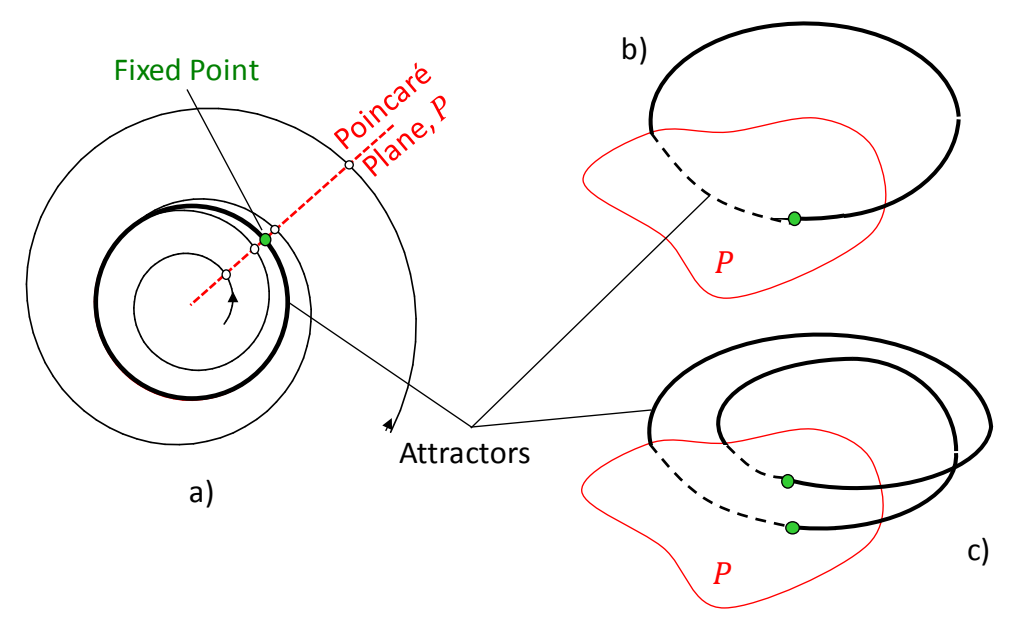

Figure 3. (a) Stroboscopic sampling of evolution trajectories; (b) single-loop attractor provides single-valued fixed point; (c) double-loop attractor provides double-valued fixed point.

Map (2.1) is known as logistic map [13] [14]. It obviously does not cover the complex turbulent picture of the medium in all nuances, but we assume that it is sufficient for the task. We justify this simplification by the following reasoning:

a) Map (2.1) describes dissipative dynamics

b) It accounts for the competition between positive and negative feedback in a simple form

c) It represents a wide class of theoretically explored and experimentally observed dynamical systems with the Feigembaum universality

d) It encompasses period-doubling bifurcations with the scaling factor $\delta_{F}=4.669 \cdots$.

e) Unlike one-dimensional continuous-time differential equations, it is flexible enough to describe topologically complex trajectories (like those shown in Figure 3(c)).

We define generic cellular charge $\chi$ as

$$
\chi=2 p-1
$$

Its domain $\chi \in[-1,1]$ is inherited from probability $p \in[0,1]$ and is not typical for charges $(q \in(-\infty,+\infty))$. We could rescale $\chi$ by using an appropriate map or equation, such as this one

$$
q=\frac{\mp 1}{\ln |\chi|} .
$$

However, since in this paper we are looking only for the roots of charge quantization, its domain size is irrelevant. Therefore, we remain with $\chi$ without further makeovers.

For mostly aesthetic reasons, we replace amplification parameter $A$ with control parameter $B$

$$
B=\frac{A}{2}-1
$$

with the corresponding changes in the domain size $A \in[1,4] \rightarrow B \in[-0.5,1]$. 
The lower end, $B=-0.5$, is a critical point. Below this point, $B<-0.5$ $(A<1)$, the medium loses amplification, feedback is pure negative, balance of forces is broken, and self-organization fails.

Initially, we study cell dynamics at different but fixed $B$-values. Later, we introduce a mechanism of vacuum self-regulation when the medium controls $B$-values by itself.

With the above introduced variables, the charge evolution is described by the iterated map

$$
\chi_{n+1}=\mathcal{L}\left(\chi_{n}, B\right)
$$

where

$$
\mathcal{L}(\chi, B)=B-(1+B) \chi^{2}
$$

is iteration function.

In any dynamical system time plays most important role. Time is meaningless without clocks. However, self-organized vacuum cells are in essence self-sustained oscillators and can be used to clock the local time by themselves. As time unit, it is natural to use iteration intervals $t=t_{n+1}-t_{n}$ between any two consecutive crossings of a charge trajectory with one of the Poincaré planes selected for this goal. If different cells operate asynchronously, each of them clocks their local time with its own pace. When the cells synchronize, interval $t$ acquires a global character and may serve as a time-standard for the entire synchronized network.

\subsection{Bifurcation Diagram. Zones of Stability. Special Points}

Quadratic maps (including logistic map) are well explored (see, for example [5][14]). For most researchers, they are particularly interesting as a paradigm of systems evolving toward chaotic dynamics. Ironically, we are more interested in the patterns of asymptotic stability, when a system asymptotically converges to some dynamically stable state.

Asymptotic stability varies with $B$. Continuous $B$-intervals where cell possess asymptotic stability we call zones of stability or just zones. On bifurcation diagram (Figure 4) which depicts fixed charge values $\chi_{\infty}$ vs. $B$, they are designated as $Z j$, where $j$ is the zone number. The diagram name came from bifurcations, the points where cell dynamics experience deep changes including changes in the attractor topology. We designate left and right bifurcations at the ends of each zone of stability respectfully as $B j L$ and $B j R$. Bifurcations are points of unstable equilibrium. At bifurcations, number of attractor loops (number of branches in the diagram) doubles. Within each zone, the system is asymptotically stable. When $B$ reaches the Feigenbaum point, $B_{F} \approx 0.786$, the system becomes chaotic.

In this paper we explore only the first three zones, $Z 1$ through $Z 3$, shown in Figure 4. We associate them respectfully with electromagnetic, weak, and strong nuclear interactions. 


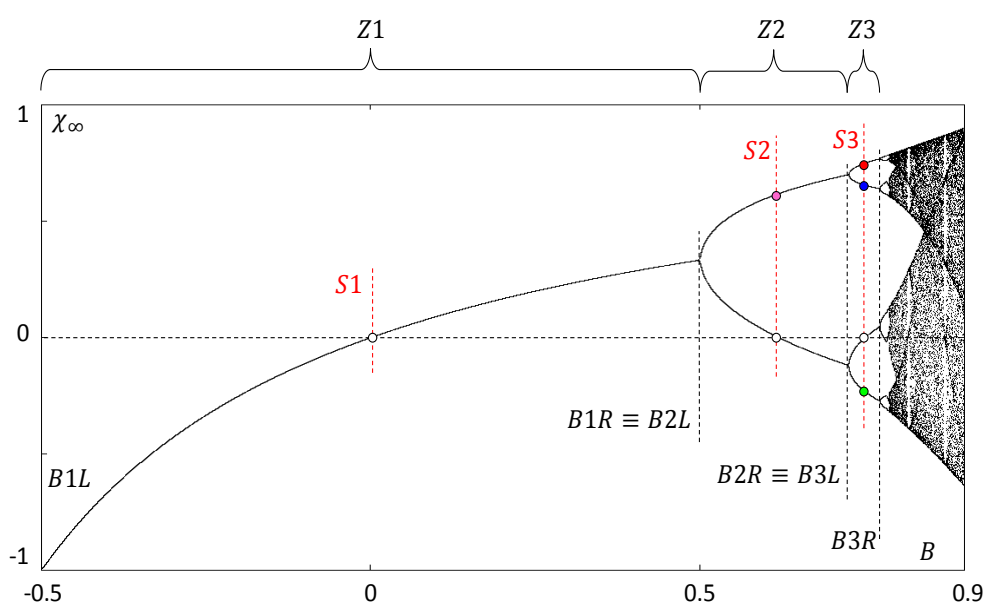

Figure 4. Bifurcation diagram depicts fixed charges $\chi_{\infty}$ as function on parameter $B$. Zones $Z j$ are marked by horizontal brackets, bifurcations $B j L$ and $B j R$ by black vertical dashed lines, superattractors $S j$ by red vertical dashed lines. Small color circles depict superattractor charges.

Later, we also explore interval $B \in(-1,-0.5)$, which we call "the dark zone" and designate as $Z 0$, where vacuum self-organization fails.

In $Z 1$, there is only one branch on the bifurcation diagram, which means that the cell attractors represent single loops, and fixed charges $\chi_{\infty}$ are singlevalued. In the higher zones, the number of branches doubles with each bifurcation, i.e. the attractors possess several loops, and fixed charges are multi-valued; $\chi_{\infty, w}, w=0,1, \cdots, \mathcal{W}_{j}-1$, where $\mathcal{W}_{j}$ is the winding number (or number of the attractor loops). For example, attractor in Figure $3(\mathrm{~b})$ has $\mathcal{W}=1$, and attractor in Figure 3(c) has $\mathcal{W}=2$.

In zone $Z j$, the winding number is

$$
\mathcal{W}_{j}=2^{j-1} \text {. }
$$

Full cell rotation period $\mathcal{T}_{j}$ comprises all different attractor loops and is

$$
\mathcal{T}_{j}=t_{j} \mathcal{W}_{j},
$$

where $t_{j}$ is the iteration time interval in zone $Z j$.

For multi-loop attractor cells with $\mathcal{W}_{j} \geq 2$ we reserve a special name. We call them spinorial cells [44]. They play a special role in toy-leptons and toy-quarks (we call them q-arcs) building blocks.

Superattractors are points of the highest asymptotic stability. We designate them as $S j$. In $Z 1$, the superattractor is located exactly at the zone center. In all other zones, superattractors are slightly shifted from the centers. In $Z 0$, the superattractor is located at the zone edge. We call intervals between superattractors $S j$ and left $B j L$ or right $B j R$ bifurcations semi-zones and designate them as $Z j L$ and $Z j R$.

Small circles at superattractors in Figure 4 depict superattractor charges. Zero charges are shown by white circles, and nonzero charges are shown by color circles (purple in $Z 2$, and red, green, and blue in $Z 3$ ).

$B$-values for special points on the bifurcation diagram are listed in Table 1 . 
Table 1. $B$-values for special points in zones $Z 1$ through $Z 3$.

\begin{tabular}{|c|c|c|c|}
\hline \multirow{2}{*}{ Special Points } & \multicolumn{3}{|c|}{$B$ values in different zones } \\
\hline & $Z 1$ & $Z 2$ & $Z 3$ \\
\hline Superattractors, $S j$ & 0 & $0.6180339887 \ldots$ & $0.7492808496 \ldots$ \\
\hline Left Bifurcations, $B j L$ & -0.5 & 0.5 & $0.7247448713 \ldots$ \\
\hline Right Bifurcations, $B j R$ & 0.5 & $0.7247448713 \ldots$ & $0.7720451797 \ldots$ \\
\hline Winding Number, $\mathcal{W}_{j}$ & 1 & 2 & 4 \\
\hline
\end{tabular}

With each bifurcation, zone size $(B j R-B j L)$ decreases approximately as geometric progression .

$$
\frac{B(j+1) R-B(j+1) L}{B j R-B j L}=\delta_{j}^{-1},
$$

where scaling factor $\delta_{j}$ quickly converges to the Feigenbaum delta $\delta_{j \rightarrow \infty} \rightarrow \delta_{F}=4.669 \cdots$

\subsection{Cellular Evolution in Z1. Uncertainty Intervals}

Z1 cells are the simplest in the model and possess single-loop attractors. In this section, we explore how the charge asymptotic stability depends on a fixed parameter $B$ in $Z 1$. We will study cell evolution toward the attractors for different $B$ values.

We assume that cells evolution may start from any initial charge value $\chi_{0}$ between 1 and -1 . A few examples of trajectories $\chi_{n}$ are shown in Figure 5. All trajectories converge toward their fixed points. Trajectories with different parameters $B$ are represented by different colors. They converge to different fixed points. However, all trajectories belonging to the same $B$-value converge to the same fixed point $\chi_{\infty}(B)$.

At the beginning of the cell evolution, the trajectories converging to one attractor entangle with trajectories converging to a different attractor. The attractors can be resolved only after some elapsing time. The longer is the elapsing time, the better is the attractor resolution.

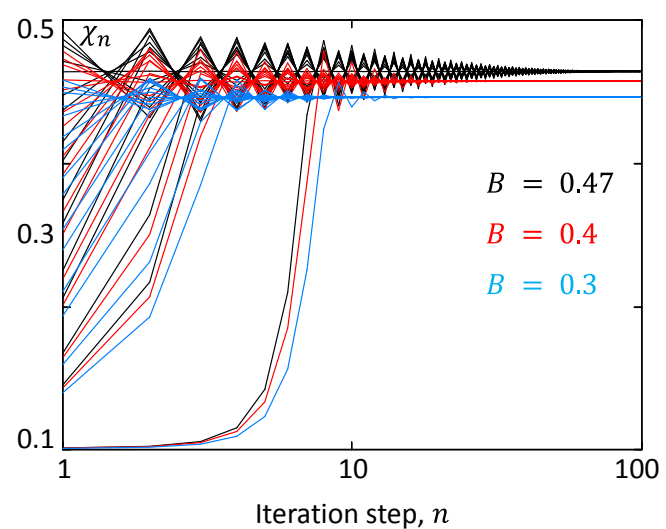

Figure 5. Charge evolution trajectories $\chi_{n}$ for three parameters $B$ (shown in three colors). 
The entanglement and asymptotic behavior of charge trajectories have a qualitative analog in quantum mechanics in a form of the time-energy uncertainty relation-the longer a system is located at some state the better the state energy (fixed point) can be discriminated. This comparison implies that quantum-mechanical eigenstates possess asymptotic stability thus quantum-mechanical systems are in essence dissipative, which is in agreement with the dissipative character of the wave-function collapses.

To quantitatively compare asymptotic stabilities of different attractors, we need to have a measure of the asymptotic stability. First, we define a charge uncertainty interval $\Delta \chi_{n}$ for a given trajectory as

$$
\Delta \chi_{n}=\left|\chi_{n}-\chi_{\infty}\right|,
$$

where $n$ is the iteration step number after beginning of the cell evolution. Then we define parameter $\lambda$ as a dimensionless slope of $\Delta \chi_{n}$ trajectory depicted in logarithmic scale

$$
\lambda=t \frac{\mathrm{d} \ln \Delta \chi}{\mathrm{d} t} .
$$

After a few iteration steps (see Figure 6), the slopes exponentially converge to their fixed values

$$
\lambda=\lambda_{\infty} \approx \lambda_{n}=\ln \Delta \chi_{n+1}-\ln \Delta \chi_{n}=\ln \frac{\Delta \chi_{n+1}}{\Delta \chi_{n}} .
$$

From Figure 6, one can see that the slopes depend only on parameter $B$.

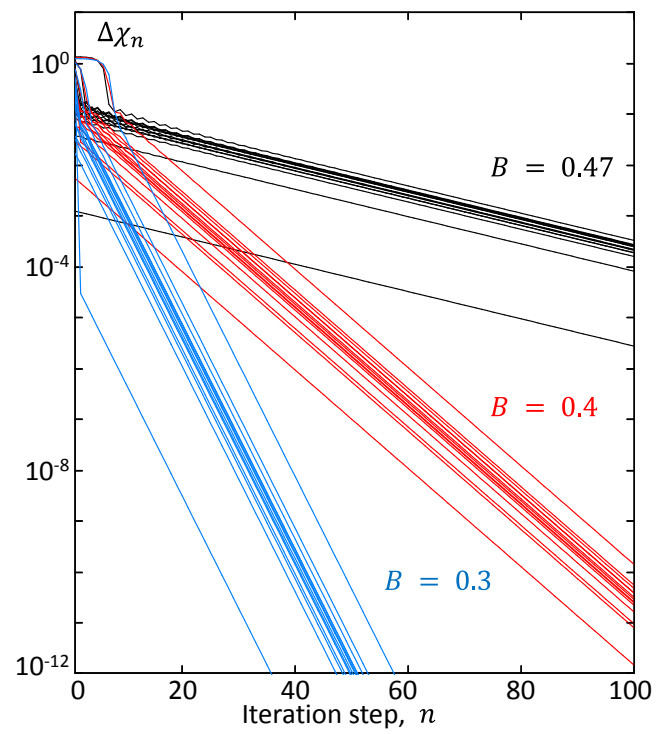

Figure 6. Evolution of uncertainty intervals $\Delta \chi_{n}$ (in logarithmic scale) for trajectories shown in Figure 5.

Slope $\lambda$ plays the role of the Lyapunov exponent. It is a measure of the cell asymptotic stability. Within the zone, it is always negative $\lambda<0$, approaches its minimum $\lambda \rightarrow-\infty$ at the super attractor, and reaches its maximum $\lambda=0$ at the zone edges (bifurcations). 


\subsection{Spinorial Cells}

We define spinorial cells as those with attractors having more than one loop $(\mathcal{W}>1)$.

They serve as particle building blocks. We encounter two types of spinorial cells, d-cells ( $d$ from double-loop attractor) with winding number $\mathcal{W}_{2}=2$ (Figure 3(c)), and q-cells ( $q$ from quadruple-loop attractor) with $\mathcal{W}_{3}=4$.

$\mathrm{D}$-cells live in zone $\mathrm{Z2}$. An example of $Z 2$-cell charge evolution trajectory is shown in Figure 7 by green seesaw line. In the limit $n \rightarrow \infty$, d-cell oscillates between two fixed values of the double-valued fixed points. We can split the single-step trajectory on two double-step trajectories, each with its own singlevalued fixed point. The odd-step trajectory $\chi_{2 n+1}$ is shown by the black dotted line. It converges toward fixed value $\chi_{\infty, \text { odd }}$. The even-step trajectory $\chi_{2 n}$ is shown by the red dotted line. It converges toward $\chi_{\infty, \text { even }}$.

For double-step trajectories we can explore the cell asymptotic stability in the same way as we did it for $Z 1$ cells.

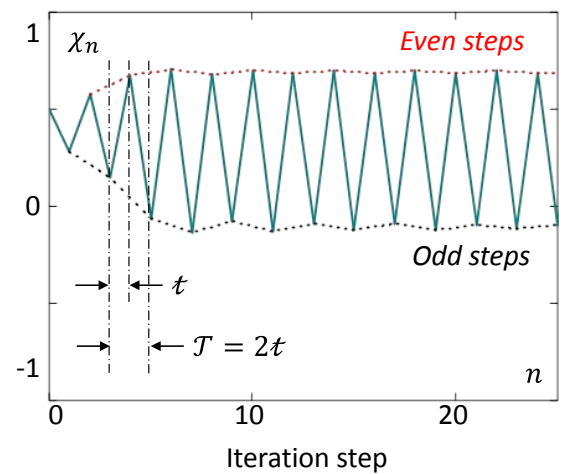

Figure 7. Example of $d$-cell charge evolution.

For each double-step trajectory, we define a charge uncertainty interval as

$$
\left\{\begin{array}{l}
\Delta \chi_{2 n}=\left|\chi_{2 n+1}-\chi_{\infty, \text { even }}\right| \\
\Delta \chi_{2 n+1}=\left|\chi_{2 n}-\chi_{\infty, \text { odd }}\right|
\end{array}\right. \text {. }
$$

Remarkably, both uncertainty intervals $\Delta \chi_{2 n+1}$ and $\Delta \chi_{2 n}$ exponentially decay with the same rates (Figure 8), i.e. with the same slopes

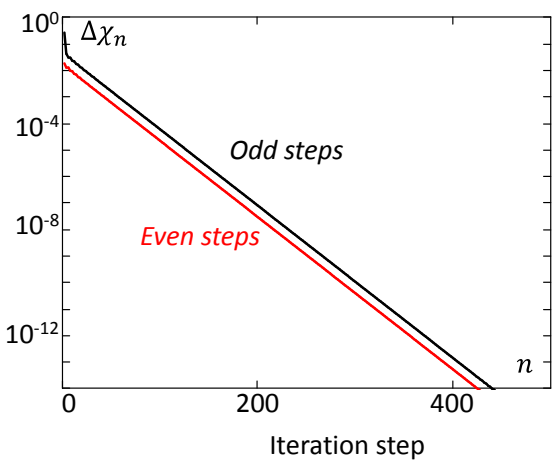

Figure 8. Example of evolution of $d$-cell uncertainty interval. 


$$
\lambda_{\text {odd }}=\lambda_{\text {even }}=\lambda .
$$

Double-step evolution trajectories are described by twice-iterated functions $\mathcal{L} 2_{\text {even }}(\chi, B)$ and $\mathcal{L} 2_{\text {odd }}(\chi, B)$ sampled at alternating time instants

$$
\left\{\begin{array}{l}
\mathcal{L} 2_{\text {even }}(\chi, B)=\mathcal{L}\left(\mathcal{L}\left(\chi_{\text {even }}, B\right)\right) \\
\mathcal{L} 2_{\text {odd }}(\chi, B)=\mathcal{L}\left(\mathcal{L}\left(\chi_{\text {odd }}, B\right)\right) \\
\chi_{0} \in(-1,1), \chi_{1}=\mathcal{L}\left(\chi_{0}\right)
\end{array}\right.
$$

Q-cells live in zone Z3. Their attractors have four loops, and their fixed points are four-valued. An example of $Z 3$-cell charge evolution trajectory is shown in Figure 9 by green seesaw line. It rotates around four dotted lines (shown in black, red, green, and blue) each depicting a four-step trajectory, $\chi_{\infty, w}$, where $w=0,1,2,3$ is the step number within a single period. The color states are ordered. The colors always come in the same sequence: $\cdots \rightarrow$ black $\rightarrow$ red $\rightarrow$ green $\rightarrow$ blue $\rightarrow$ black $\rightarrow \cdots$. Due to the time irreversibility this order cannot be inverted. This order and irreversibility are crucial in discussion of quark (or more correctly, q-arc) mixing angles.

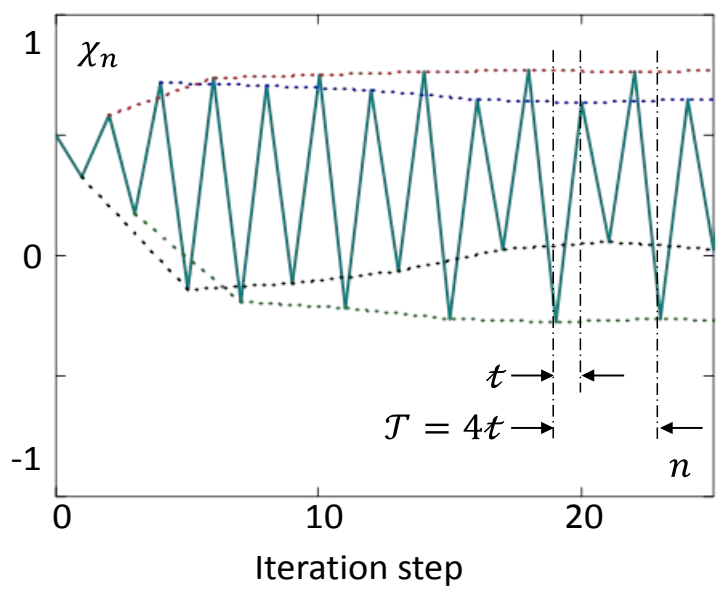

Figure 9. Example of q-cell charge evolution.

Four-step charge uncertainty intervals $\Delta \chi_{n, w}$ are defined as

$$
\Delta \chi_{n, w}=\left|\chi_{n, w}-\chi_{\infty, w}\right|, \quad w=0,1,2,3,
$$

Their typical evolution is shown in Figure 10. As in the previous case, all of them have the same slopes $\lambda_{w}=\lambda$.

Four-step iteration functions $\mathcal{L} 3_{n, w}$ are four-times iterated the original function $\mathcal{L}_{n}$ sampled at different time instants

$$
\left\{\begin{array}{l}
\mathcal{L} 3_{n, w}=\mathcal{L} 2\left(\mathcal{L} 2\left(\chi_{n, w}, B\right)\right)=\mathcal{L}\left(\mathcal{L}\left(\mathcal{L}\left(\mathcal{L}\left(\chi_{n, w}, B\right)\right)\right)\right) \\
\chi_{0,0} \in(-1,1), \chi_{0, w}=\mathcal{L}\left(\chi_{0, w-1}\right)
\end{array}\right.
$$

As in the previous case, initial charge $\chi_{0,0}$ of the first trajectory can be chosen arbitrarily, while the initial charges of the other trajectories $\chi_{0, w}$ are images of $\chi_{0,0}$ after the corresponding number of iterations. 


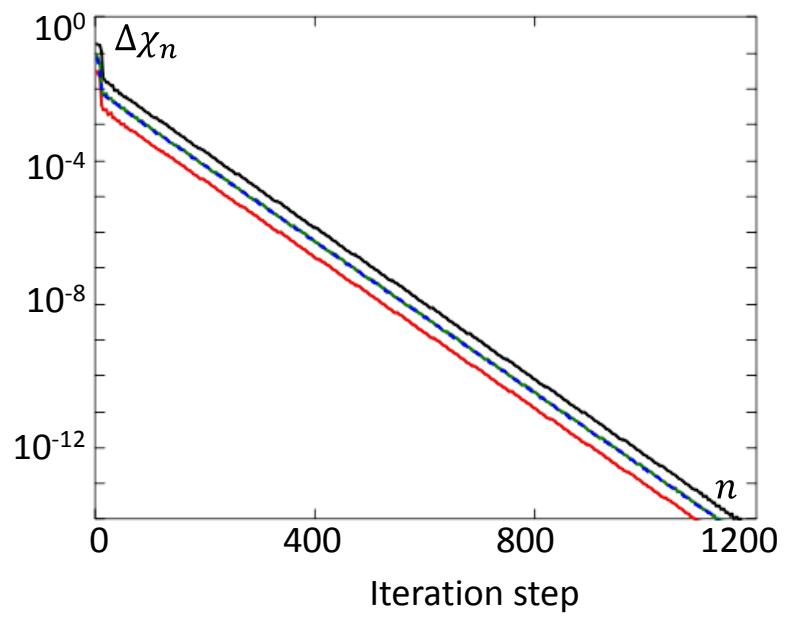

Figure 10. Example of evolution of q-cell uncertainty interval.

The multi-step trajectories have single-valued fixed points and are more suitable for analysis. Examples of $\mathcal{L} 1(\chi), \mathcal{L} 2(\chi)$ and $\mathcal{L} 3(\chi)$, for a randomly selected $B$-values from each of $Z 1, Z 2$, and $Z 3$ zones, are shown in Figure 11.

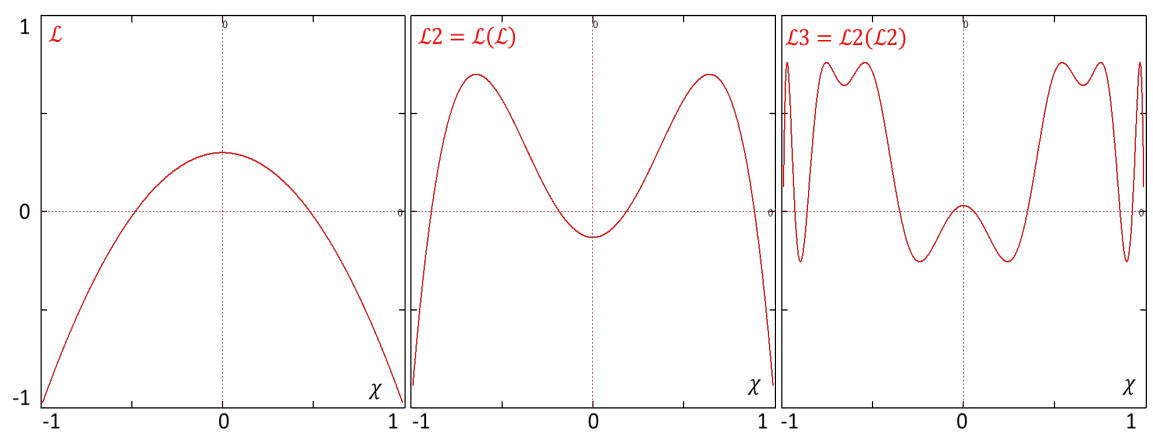

Figure 11. Examples of $\mathcal{L} 1(\chi), \quad \mathcal{L} 2(\chi)$ and $\mathcal{L} 3(\chi)$ for randomly selected $B$ values.

In each semi-zone, asymptotic stability depends on how far the function local extrema located from the super attractor in $B$-space. We define the distance between the cell attractor $(B)$ and the superattractor $S j$ as

$$
\Delta B=B-S j .
$$

Because different semi-zones have different sizes, to compare asymptotic stability in different zones, we use reduced distance $\beta$, which we define as

$$
\beta=\left\{\begin{array}{l}
\frac{\Delta B}{|B j L-S j|} \text { for } Z j L \\
\frac{\Delta B}{|B j R-S j|} \text { for } Z j R
\end{array}\right.
$$

where the denominator is the size of the corresponding semi-zone, $j$ is the zone number, $S j$ is superattractor, $B j L$ is the left bifurcation, and $B j R$ is the right bifurcation. The reduced distance domain is the same in all zones $\beta \in[-1,1]$. For all superattractors $\beta=0$, and for bifurcations $\beta= \pm 1$. 
Before comparing asymptotic stabilities in different zones, we also need to bring them to the same time scale. We assume, that after cells become synchronized, they all have the same period $\mathcal{T}$

$$
\mathcal{T}=\mathcal{T}_{j}, j=1,2, \cdots
$$

Correspondingly, their iteration intervals in zones $Z j$ are

$$
t_{j}=\frac{\mathcal{T}}{\mathcal{W}_{j}} .
$$

With these assumptions, we extend definition of parameter $\lambda$ given by (2.11) for all zones $Z j$ as

$$
\lambda=\mathcal{T} \frac{\mathrm{d} \ln \Delta \chi}{\mathrm{d} t}
$$

After a few iteration steps (see Figure 10) the trajectories almost reach their fixed points and the slopes can be determined as

$$
\lambda \approx \ln \Delta \chi_{n+w_{j}}-\ln \Delta \chi_{n}=\ln \frac{\Delta \chi_{n+w_{j}}}{\Delta \chi_{n}} .
$$

Simulated dependences $\lambda(|\beta|)$ in six semi-zones, from $Z 1 L$ through $Z 3 R$, are shown in Figure 12 by different symbols. All six are similar to each other and can be approximated as a logarithmic function (red solid curve)

$$
\lambda=\ln |\beta| .
$$

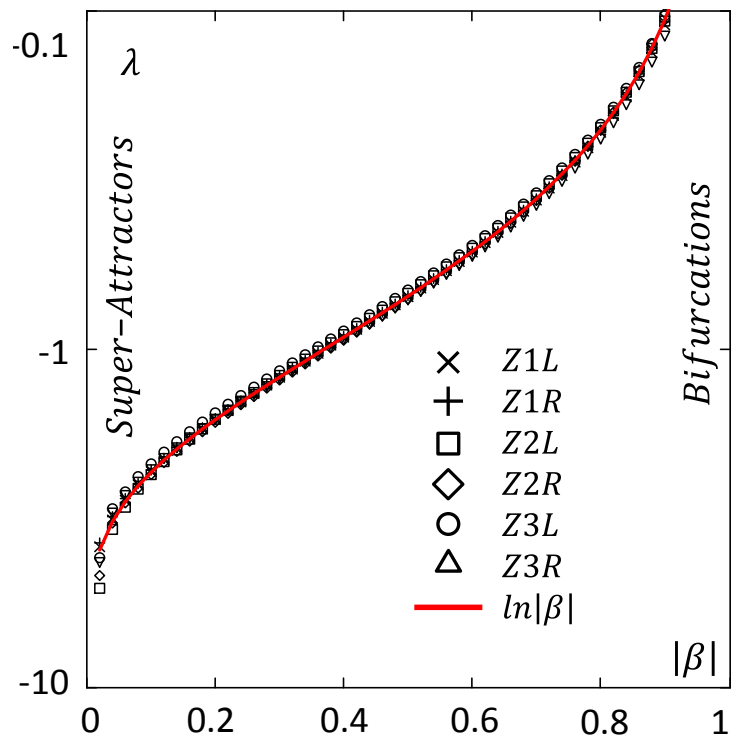

Figure 12. Calculated functions $\lambda(|\beta|)$ for six semi-zones ( $Z 1 L$ through $Z 3 R$ ) are represented by different symbols. Solid red line depicts function $\ln |\beta|$.

\subsection{Energy and Chemical Potentials}

We define cell energy as 


$$
\mathcal{E}=\frac{\lambda}{\mathcal{T}}
$$

$\mathcal{E}$ has physical dimensions of frequency. It inherits many features of parameter $\lambda$. For example, we can use it as a measure of cell asymptotic stability. Excluding bifurcations, where $\mathcal{E}=0$, it is always negative and takes the most negative values at the superattractors. It is customary to associate negative energies with the bound states. In our case, a vacuum cell is bound by the dust selfattraction forces.

Using (2.24) and (2.25), we obtain that energy depends on parameter $\beta$ as

$$
\mathcal{E}(\beta)=\frac{1}{\mathcal{T}} \ln |\beta|
$$

If we neglect the small differences between the left and right semi-zones, from (2.9) we obtain that

$$
\beta \approx 2 \delta_{F}^{j-1} \Delta B .
$$

From (2.26) and (2.27), we obtain that energy depends on $\Delta B$ in all zones as

$$
\mathcal{E}(\Delta B, j) \approx \frac{1}{\mathcal{T}} \ln \left|2 \delta_{F}^{j-1} \Delta B\right|=\mathcal{E}(\Delta B)+\mu_{j}=\mathcal{E}(B-S j)+\mu_{j} .
$$

where $\mathcal{E}(\Delta B) \equiv \mathcal{E}(\Delta B, 1)$, and $\mu_{j}$ is a parameter that we associate with chemical potential (internal cellular energy) in the corresponding zone and is defined as

$$
\mu_{j}=\frac{1}{\mathcal{T}}(j-1) \ln \delta_{F} .
$$

Cellular energy $\mathcal{E}$ as function on parameter $B$ is shown in Figure 13. It has a form of three connected potential wells, each covering its own zone, and having deep minima at the superattractors.

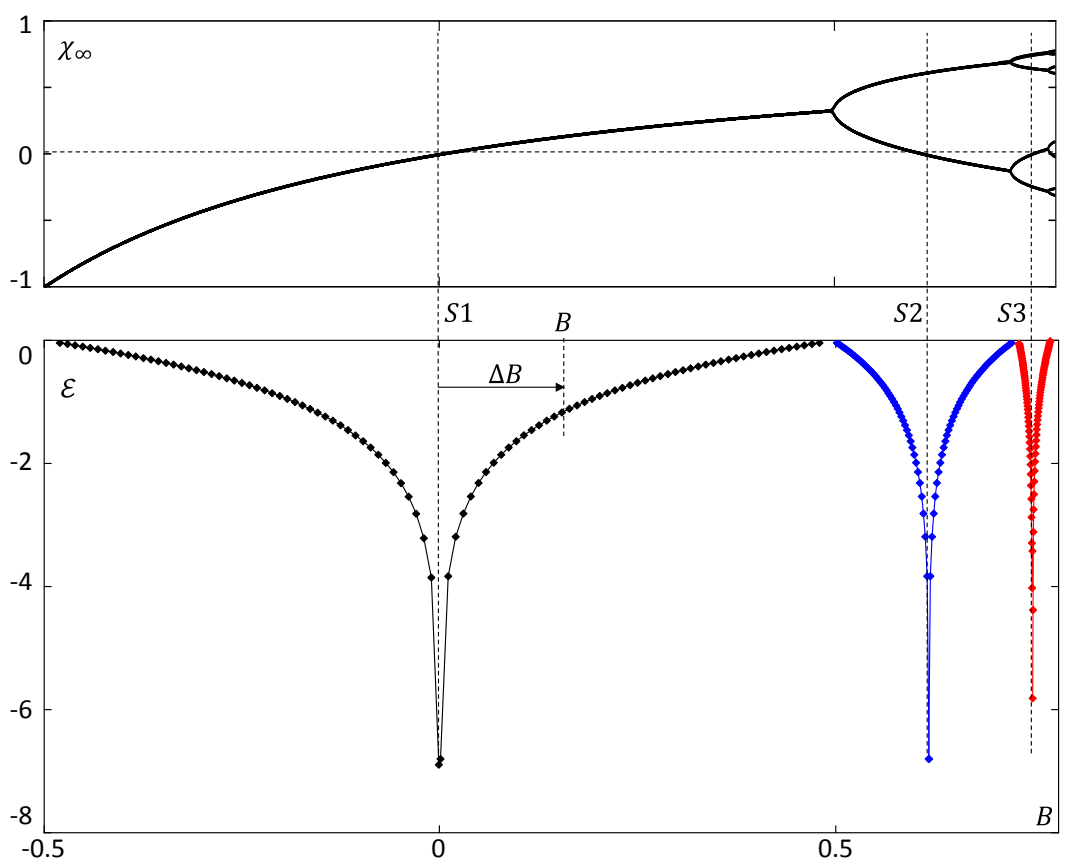

Figure 13. $\mathcal{E}(B)$ in $Z 1$ through $Z 3$ (bottom) and bifurcation diagram (top). 
The well shapes are almost identical. $Z 2$-well and $Z 3$-well can be obtained from $Z 1$-well by horizontal and vertical translations (see (2.30)) as it is shown in Figure 14. Vertical shifts are equal to the chemical potential differences in the corresponding zones.

$$
Z 1 \rightarrow Z j=\left\{\begin{array}{l}
S 1 \rightarrow S j \\
\mathcal{E} \rightarrow \mathcal{E}+\mu_{j}
\end{array}\right.
$$

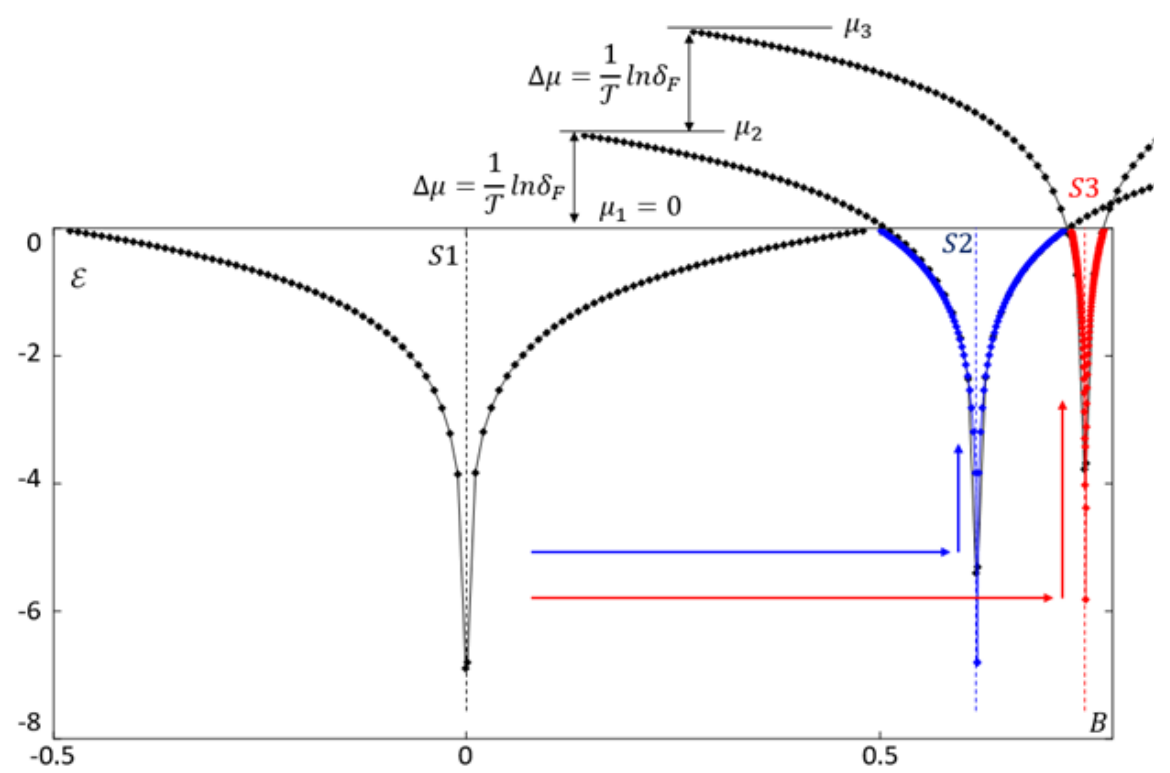

Figure 14. Directly calculated $Z 2$-well (blue) and $Z 3$-well (red), and translated copies of $Z 1$-well (black) according to (2.30).

\subsection{Partition Function. Vacuum Self-Regulation. Discrete State Spectrum}

In this section we introduce a vacuum self-regulation mechanism. It controls cell distribution among the attractors.

We assume that during their evolution, charge trajectories converging to a given attractor do not disappear. They squeeze together during cell evolution, and their density $\rho$ increases with elapsing time in inverse proportion to the uncertainty intervals

$$
\rho(t, \mathcal{E})=\frac{\rho_{0}}{\Delta \chi(t, \mathcal{E})},
$$

where $\rho_{0}$ is initial trajectory density, and uncertainty intervals $\Delta \chi(t, \mathcal{E})$ after a few iteration steps can be described as

$$
\Delta \chi(t, \mathcal{E}) \propto \exp (\mathcal{E} t)
$$

Being an open system, a vacuum cell, under influences of random external forces, occasionally jumps from one trajectory to another. We define vacuum temperature $\theta$ as average frequency of these jumps

$$
\theta=\frac{1}{t_{\theta}}
$$


where $t_{\theta}$ is the average dwell time at a single trajectory.

We assume also that a jumping cell has equal probabilities to "land" onto any trajectory.

With the above assumptions, the probability $w$ to find a cell near attractor with energy $\mathcal{E}$ is proportional to the average trajectory density $\rho(t, \mathcal{E})$

$$
w(t, \mathcal{E}) \propto \rho(t, \mathcal{E}) \propto \exp (-\mathcal{E} t) .
$$

By combining (2.33) and (2.34), and replacing $t \rightarrow t_{\theta}$, we obtain that in zone $Z 1$ at temperature $\theta$, partition function is

$$
w(\mathcal{E}, \theta)=C_{0} \exp \left(-\frac{\mathcal{E}}{\theta}\right)
$$

where $C_{0}$ is a normalization constant. Using (2.28) and (2.29), we can extent (2.35) to the other zones

$$
w_{j}(\mathcal{E}, \theta)=C_{0} \exp \left(-\frac{\mathcal{E}+\mu_{j}}{\theta}\right)
$$

where $j$ is the zone number, and $\mu_{j}$ is the corresponding chemical potential.

Distribution (2.36) is the equation of vacuum self-regulation. It provides the probability to find a cell near the corresponding attractor (energy). Its form remarkably resembles that of the Boltzmann factor. The major difference is that the latter normally provides distribution of free particles (positive energies), while partition function (2.36) controls distribution of bounded cells (negative energies).

Partition function (2.36) can be rewritten for cell distribution in $B$-space. Three examples of $w(B)$ are shown in Figure 15 for three different temperatures (dwell times $t_{\theta}$ ). The curves are normalized as

$$
w\left(t_{\theta}, B\right)=\frac{\Delta \chi\left(t_{\theta}, B\right)^{-1}}{\left[\Delta \chi\left(t_{\theta}, B\right)^{-1}\right]_{\max }}
$$

At very high temperatures, when cell dwell time at a single trajectory decreases down to the length of the iteration interval $t_{\theta} \sim t$, the distributions acquires form of a continuous spectrum (blue and green curves in Figure 15). At lower temperatures, dwell time $t_{\theta} \gg t$ is large enough for a cell to make a few rotations before it jumps to another trajectory, the partition function acquires the shape of the Dirac $\delta$-function (red curve), and the cell distribution spectrum becomes essentially discrete.

At normal temperatures, the vast majority of cells are located at the superattractors, and the superattractor characteristics acquire rank of physical constants. They are not absolute but may deviate from the superattractor values under action of external forces and temperature, i.e. these constants are "running". (Recall the running coupling constants in the standard model, like electron charge). 


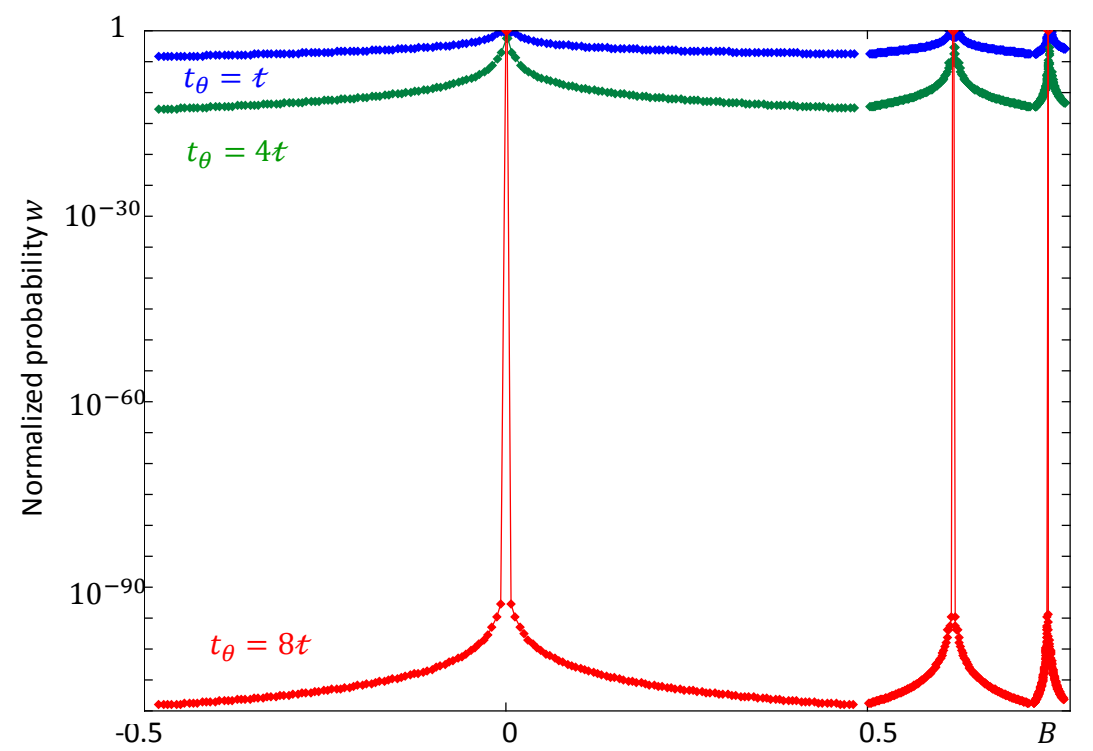

Figure 15. Normalized cell distributions among attractors $(B)$ at temperatures $\theta=t^{-1} ; 0.25 t^{-1}$, and $0.125 t^{-1}$.

\subsection{Chemical Potentials and Electroweak Mixing Angles}

Let us return to very high temperatures, when cellular jumps among the trajectories become so frequent, that cells do not have enough time to complete even a single rotation. At these temperatures, cells lose their cellular features and dissolve in the background dust. The critical temperature of the cellular "melting" is

$$
\theta_{c} \sim \frac{1}{\mathcal{T}},
$$

where $\mathcal{T}$ is the cell rotation period.

If "melted" vacuum cools down to the temperatures below critical, $\theta_{c}$, cellular formation starts again. Vacuum can produce cells of different types that belong to different zones of stability. The number of cells of each type depends on the corresponding chemical potential $\mu_{j}$.

Let us illustrate this on a simple example, when the resulting cell mixture consists only of two types of cells, $Z 1$-cells and $Z 2$-cells. According to our associations with the standard model, these cells represent respectfully electromagnetic interactions and weak nuclear interactions. We want to estimate their relative input, i.e. ratio $w_{Z 2} / w_{Z 1}$, where $w_{Z 1}$ and $w_{Z 2}$ are probabilities to find respectively $Z 1$-cells and $Z 2$-cells. In a two-component mixture, the total probability is

$$
w_{Z 1}+w_{Z 2}=1 .
$$

We can replace $w_{Z 1}$ and $w_{z 2}$ with trigonometric functions of a single parameter, angle $\gamma$, which we call a mixing angle

$$
\left\{\begin{array}{l}
\cos ^{2} \gamma+\sin ^{2} \gamma=1 \\
w_{Z 1}=\cos ^{2} \gamma \\
w_{Z 2}=\sin ^{2} \gamma
\end{array}\right.
$$

Let us assume that at the melting point the temperature is fixed and is $\theta_{c}$. 
Then, using (2.29) and (2.36), one can find that the $w_{Z 2} / w_{Z 1}$ is also fixed and is

$$
\frac{w_{Z 2}}{w_{Z 1}}=\exp \left(\frac{\mu_{1}-\mu_{2}}{\theta_{c}}\right)=\delta_{F}^{-1} \approx 0.214 \text {, }
$$

Here $\mu_{1}=0, \mu_{2}=\frac{1}{\mathcal{T}} \ln \delta_{F}$, and $\delta_{F}$ is the Feigenbaum delta.

Using (2.40) and (2.41), one can find that the mixing angle $\gamma$ is also fixed and is

$$
\gamma=\operatorname{atan}\left(\sqrt{\frac{w_{Z 2}}{w_{Z 1}}}\right) \approx 24.8^{\circ}
$$

Recall, that in the standard model, there exists a fixed number $\theta_{W} \approx 28.7^{\circ}$, which value is close to $\gamma$, which also calls "mixing angle" (the Weinberg mixing angle or electroweak mixing angle), because it provides the relative input of electromagnetic ( $B^{0}$-boson) and weak ( $W^{\oplus}$-boson) components in their mixture that calls photon.

The Weinberg mixing angle is an empirical constant. It is connected to the electromagnetic and weak interaction coupling constants $\alpha$ and $\alpha_{w}$ via Equation (recall (1.8))

$$
\sin ^{2} \theta_{W}=\frac{\alpha}{\alpha_{w}} \approx \delta_{F}^{-1} .
$$

By comparing (1.8) and (2.41), one can deduce that the coupling constants can be expressed via cellular chemical potentials

$$
\frac{\alpha}{\alpha_{w}} \approx \frac{w_{Z 2}}{w_{Z 1}}=\exp \left(\frac{\mu_{1}-\mu_{2}}{\theta_{c}}\right) \text {. }
$$

This remarkable result suggests that the standard model coupling constants are thermodynamical characteristics of the corresponding fields.

Extending the model to three-component mixture is straightforward. Adding $Z 3$-cells associated with the strong nuclear interactions, and including the strong coupling constant $\alpha_{s}$ in (2.43) gives us the proportion

$$
\alpha: \alpha_{w}: \alpha_{s} \approx w_{Z 3}: w_{Z 2}: w_{Z 1}=\exp \left(\frac{\mu_{1}}{\theta_{c}}\right): \exp \left(\frac{\mu_{2}}{\theta_{c}}\right): \exp \left(\frac{\mu_{3}}{\theta_{c}}\right) \approx 1: \delta_{F}: \delta_{F}^{2},
$$

that is in agreement with(1.13)!

\subsection{Anti-Cells}

Charge conjugation is the only component of CPT-symmetry, which has an analog in the proposed model.

If in a given cell we invert directions of all radial flows, we obtain a complimentary charged cell. In the standard model, anti-particles can be represented as particles evolving backward in time. This possibility is inherited from Hamiltonian framework where time reversibility is one of the fundamental symmetries. In dissipative framework where the proposed model belongs to, we cannot revert time direction. This would convert all attractors into repellers and instead of asymptotic stability we obtain chaos. To invert intracellular flow directions without reversing time direction, we will use specific features of the even itera- 
tion function. To describe anti-cells, we will replace the original iteration function with its negative copy

$$
\mathcal{L}(\chi, B) \equiv-\mathcal{L}(\chi, B) .
$$

The inverted map (2.45) evolves in the same time direction. It possesses all features of the original map (2.5). One can find exactly the same special points at the same places, the same parameter values for $\lambda, \beta, \mathcal{E}$ and $\mu_{j}$, the same relations among them, the same levels of the asymptotic stability, the same probability distributions, etc. The only difference is that all intracellular flows are reverted and all charges have the opposite polarities.

The combined cell/anti-cell iteration function is

$$
\mathcal{L}^{ \pm}= \pm\left[B-(1+B) \chi^{2}\right]
$$

where sign “+” stands for cells, and “-” for anti-cells.

The combined cell/anti-cell bifurcation diagram is shown in Figure 16. It comprises the original diagram that depicts fixed charge values for cells (shown in black) and the inverted diagram that depicts fixed charge values for anti-cells (shown in red).

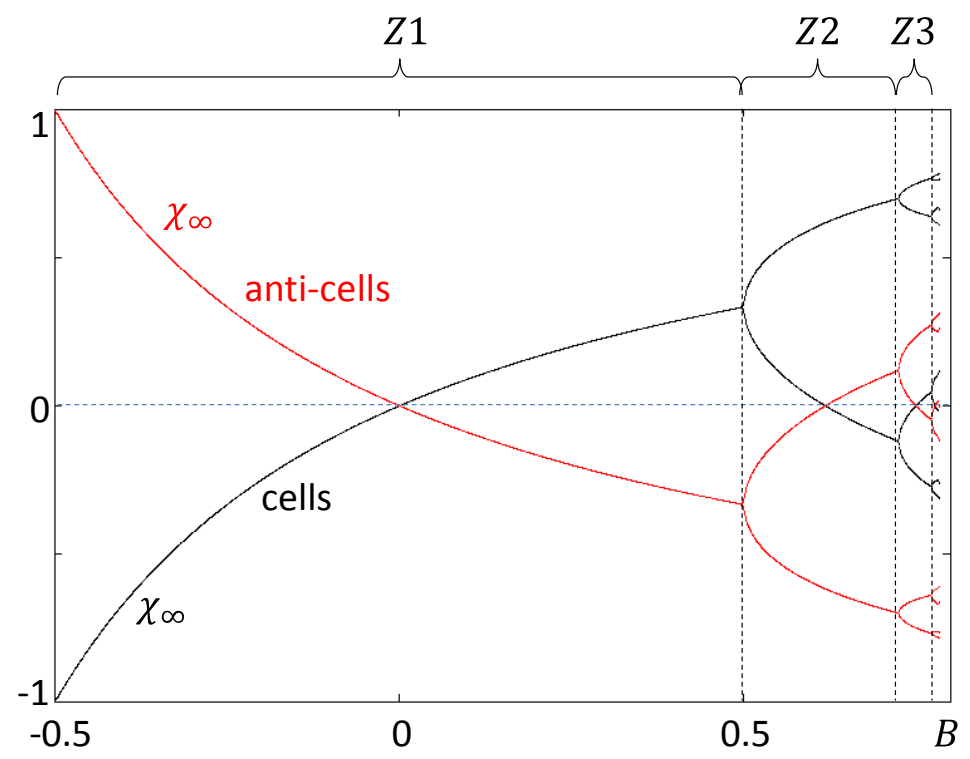

Figure 16. Combined cell/anti-cell bifurcation diagram.

\subsection{Discrete Cell Distribution Implies Quantized Charges}

In section 2.6, we found that, for not extremely hot vacuum, cell distribution among the attractors is practically discrete. Almost all the cells are located near the superattractors, and superattractor characteristics become physical constants. The superattractor charges are among these special numbers. The charged superattractor states are shown on the bifurcation diagram in Figure 17 by small color circles. Each charged state has its counterpart (the anti-state with the opposite charge polarity). Beside the charged superattractor states there exist neutral superattractor states. They are shown by white circles. 


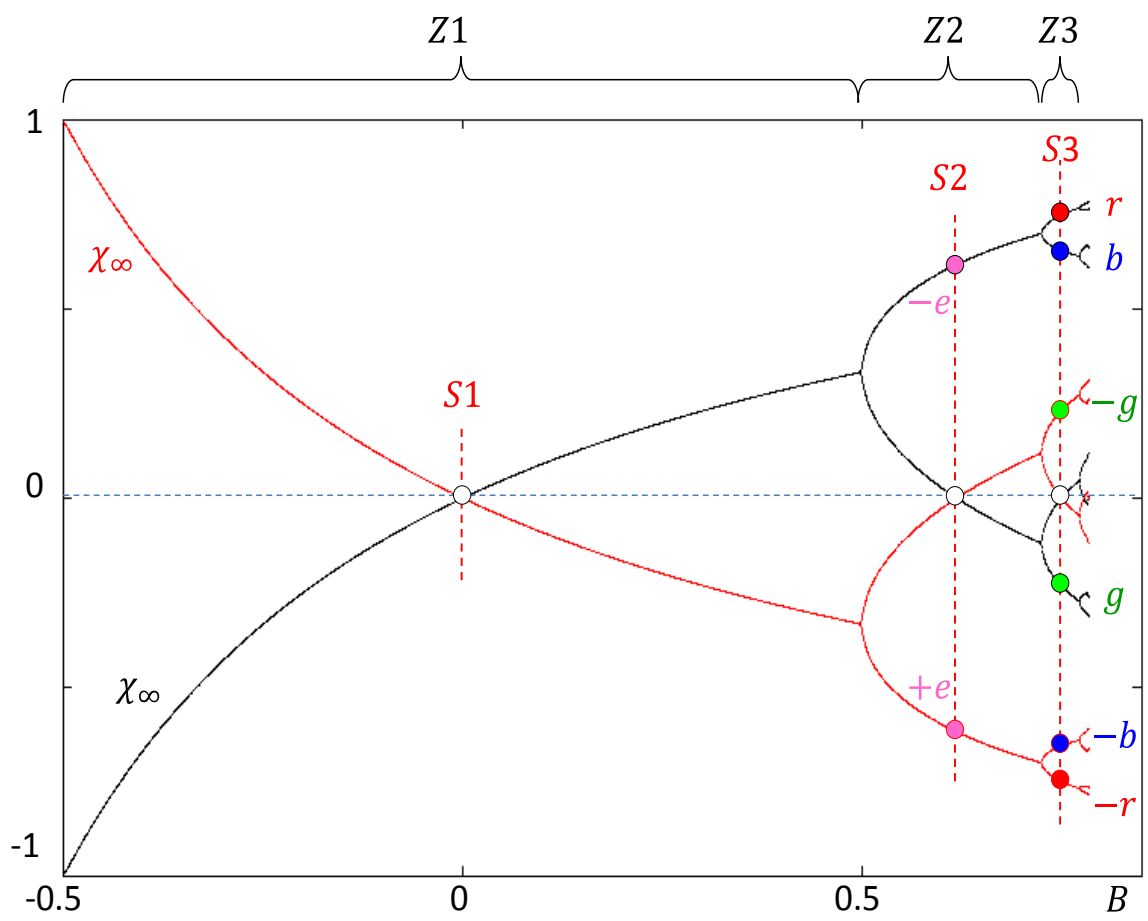

Figure 17. Quantized charges of the proposed model are shown by color circles.

In zone $Z 1$, which we associate with electromagnetic field, the only superattractor state is neutral, $\chi_{S 1, \infty}=0$, and this is in agreement with electrically neutral photons.

In zone $Z 2$, which we associate with weak nuclear field, there exist one neutral superattractor state and two complimentary charged states (purple circles). The charged states are in agreement with weak charge spectrum. Recall that in weak interactions, there exist neutral mediators, $W^{0}$ boson, and charged mediators, $W^{ \pm}$bosons, carrying charges $\pm e$.

In zone $\mathrm{Z3}$, which we associate with strong nuclear field, there exist one neutral state and three pairs of complimentary charged states shown by red, green, and blue circles. The charged states are in agreement with the color charge spectrum carried by gluons, and we can associate red, green, and blue superattractor charges with color charges of chromodynamics. Z3 neutral state has no analog in the standard model, but it does play an important role in explanation of flavor mixing amplitudes.

One of the spinorial cell peculiarities is that their charged states are dynamical: if at one time instant, $d$-cell is at the neutral (white) state, the next instant, it is at the charged (purple) state. The charge pulsations can be leveled at some distance from the cells if we couple two d-cells and synchronize them out-of-phase. Then, when one of them is charged the other is neutral and vice versa. Another peculiarity is that in q-cells the charge states rotate always in the same order: ... $\rightarrow$ neutral $\rightarrow$ red $\rightarrow$ green $\rightarrow$ blue $\rightarrow$ neutral $\rightarrow \ldots$

Some important cellular quantum numbers we have encountered in the previous discussion are summarized in Table 2. 
Table 2. Some important cellular quantum numbers.

\begin{tabular}{|c|c|c|c|c|}
\hline Zone & Associated Field & Topology & Charges & Chemical Potentials \\
\hline$Z 1$ & Electromagnetic & $\mathcal{W}_{1}=1$ & None & $\mu_{1}=0$ \\
\hline$Z 2$ & Weak Nuclear & $\mathcal{W}_{2}=2$ & $\pm e$ & $\mu_{2}=\mathcal{T}^{-1} \ln \delta_{F}=\theta_{c} \ln \delta_{F}$ \\
\hline Z3 & Strong Nuclear & $\mathcal{W}_{3}=4$ & \pm red, green, blue & $\mu_{3}=2 \mathcal{T}^{-1} \ln \delta_{F}=2 \theta_{c} \ln \delta_{F}$ \\
\hline \multicolumn{5}{|c|}{ Cell formation probabilities (cell mixing numbers) } \\
\hline \multicolumn{5}{|c|}{$w_{z 3}: w_{z 2}: w_{z 1} \approx \alpha: \alpha_{w}: \alpha_{s} \approx 1: \delta_{F}: \delta_{F}^{2}$} \\
\hline & Critical ten & rature of cell & issolution/formatio & $\theta_{c} \sim \mathcal{T}^{-1}$ \\
\hline
\end{tabular}

\section{Cellular Networks}

In Section 2, we studied individual vacuum cells. In this part, we explore their networks.

\subsection{Intercellular Dynamics. Synchronization between Two Cells}

Synchronization is a ubiquitous phenomenon in self-organized systems [39] [40] [41] [42]. Since Huygens's discovery in 1665, synchronization was observed in a number of active dissipative systems: coupled mechanical clocks, chemical reactions, electric and electronic circuits, animated cells, organs, and organisms, eco-systems, etc. Synchronization may happen between identical oscillators and between unlike devices, between couples and among components of a complex system, between oscillators producing near-sinusoidal waveforms and between chaos generators.

Vacuum cells are open systems and may couple to each other via dust exchanges as schematically shown in Figure 18. As self-sustained oscillators, they may synchronize. Synchronization is a dissipative process that occurs under repetition of delayed feedback loops. It is a phase transition that transforms independent vacuum cells into coherent networks. After synchronization, most of cells operate with one unified frequency (small amount of cells oscillate at different but commensurate frequencies). An important feature of synchronization is its universality: synchronized oscillators may differ among themselves by construction, geometry, topology, etc. This means that to synchronize, vacuum cells are not required to be identical. Below, we explore consequences of cell synchronization.

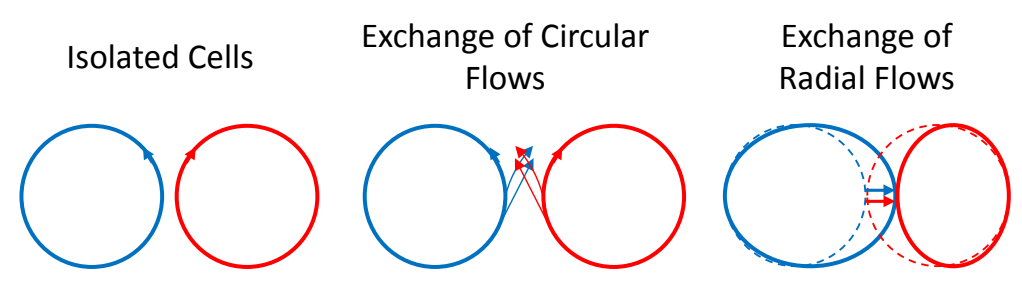

Figure 18. Cell coupling via flow exchanges. 
We start with a simple system comprising two coupled phase oscillators, which prior to synchronization operated with different natural frequencies $\omega_{1}$ and $\omega_{2}$. Each oscillator imposes a periodical force onto the other oscillator. In a simplest case, the forces are sinusoidal. The strength of the force at each time instant depends on the current phase difference between the oscillators, $\psi=\varphi_{2}-\varphi_{1}$. To describe phase dynamics, we use the Adler equation [39]:

$$
\frac{\mathrm{d} \psi}{\mathrm{d} t}=-\delta \omega+K \sin \psi,
$$

where $\delta \omega=\omega_{2}-\omega_{1}$ is initial frequency difference, and $K$ is a coupling parameter. We adapt this equation to discrete-time dynamics and transform it to the form

$$
\psi_{n+1}-\psi_{n}=-\delta \omega t+K t \sin \psi_{n},
$$

where $\psi_{n}$ is the phase difference immediately after $n$-th iteration step, and $t=t_{n+1}-t_{n}$ is the iteration time interval. To simplify notations, we set $t=1$, and obtain iterated map

$$
\psi_{n+1}=\psi_{n}-\delta \omega+K \sin \psi_{n} .
$$

If the oscillators have different natural frequencies and there is no coupling between them, the phase difference $\psi$ grows without limitation. When coupling is strong enough, the phase difference $\psi$ may converge to a fixed point $\psi \rightarrow \psi_{\infty}$. The phenomenon is known as phase entrainment or phase locking.

Map (3.3) possesses asymptotic stability $\psi_{n} \rightarrow \psi_{\infty}$ if initial frequency difference $|\delta \omega|<K$ (Figure 19 central part). Otherwise, the map is diverging $\psi_{n} \rightarrow \infty$.

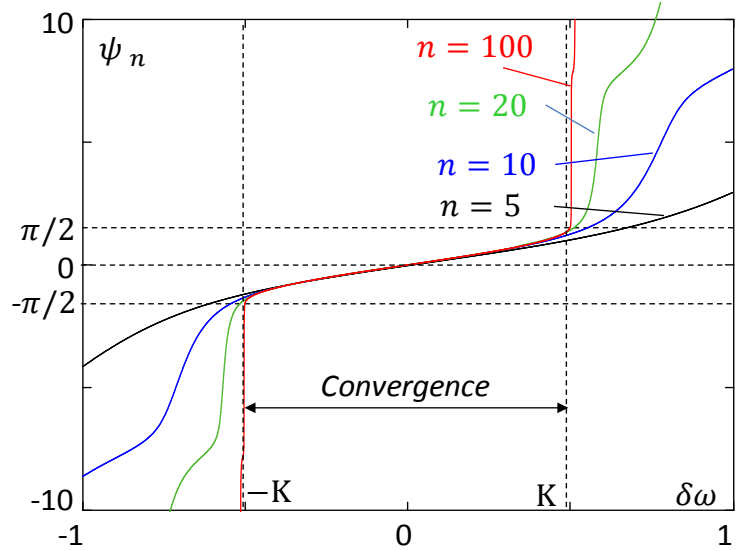

Figure 19. Phase differences $\psi_{n}$ for map (3.3) after $n$ iteration steps.

A few examples of converging $\psi_{n}$ trajectories are shown in Figure 20. Independent on initial phase difference $\psi_{0}$, the trajectories converge to fixed values, which depends on the initial frequency difference $\psi_{\infty}(\delta \omega)$. Trajectories belonging to different $\delta \omega$ are shown in different colors.

Phase-difference $(\psi)$ dynamics are similar to charge $(\chi)$ dynamics, which we studied in Section 2. At the beginning of evolution, $\psi_{n}$ trajectories belonging to different $\delta \omega$ entangle and are resolved only after some elapsing time. The 
uncertainty intervals $\Delta \psi_{n}=\left|\psi_{n}-\psi_{\infty}\right|$ shrink with time exponentially (Figure 21). Their slopes $\lambda$ depend on the initial frequency difference $\delta \omega$ and coupling $K$. The highest convergence rate occurs at superattractor $\delta \omega=0$. If $\delta \omega$ is fixed, the highest convergence rate occurs when $|K|=1$.

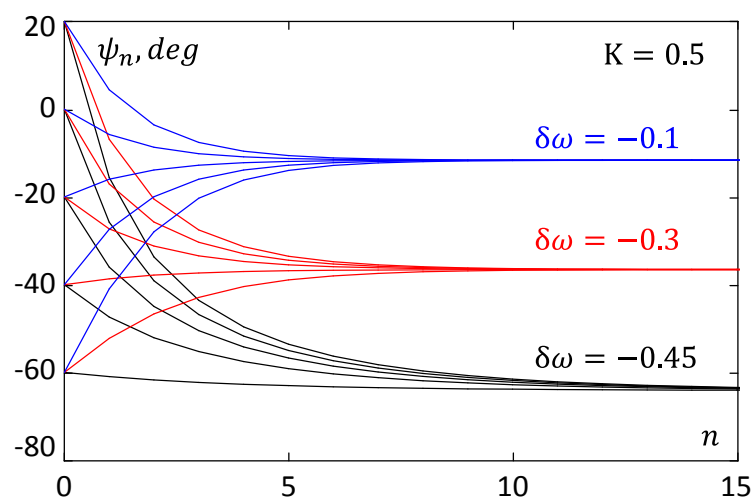

Figure 20. Examples of phase difference $\psi_{n}$ evolution. Trajectories with different $\delta \omega$ -values are shown by different colors.

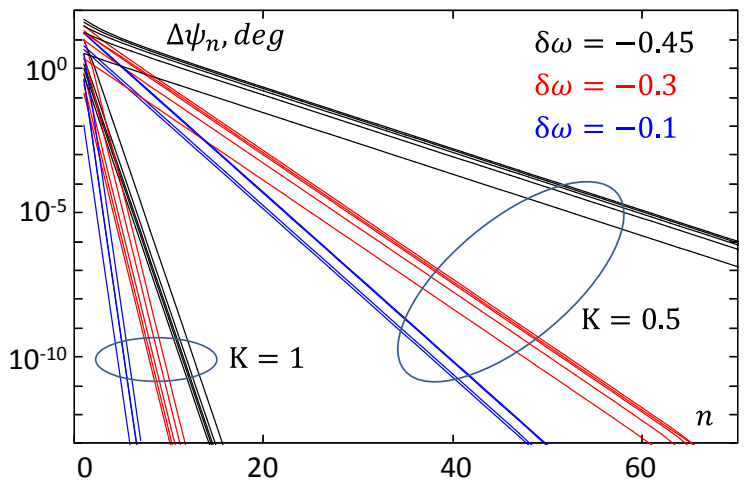

Figure 21. Examples of uncertainty interval $\Delta \psi_{n}$ evolution.

After synchronization, both oscillators operate at the same frequency. The frequency difference is always zero and $\delta \omega$ does not provide much information about oscillators. This function is "delegated" to a new order parameter, fixed points $\psi_{\infty}$

$$
\delta \omega \stackrel{\text { Sync }}{\Rightarrow} \psi_{\infty}
$$

We define energy $\mathcal{E}^{\prime}$ similarly to how we define it for a single cell (Equation (2.25)), but instead of the cell period we use frequency $\omega$ which is established after synchronization

$$
\mathcal{E}^{\prime} \equiv \lambda \omega
$$

Function $\mathcal{E}^{\prime}(\psi)$ has form of a potential well (Figure 22). The well depth depends on coupling $K$. For stronger couplings, the well deepens and its walls gradually acquire logarithmic shape (Figure 22(left)). For small couplings, the well bottom is smooth and its shape more resembles cosine function (Figure 22(right)). 

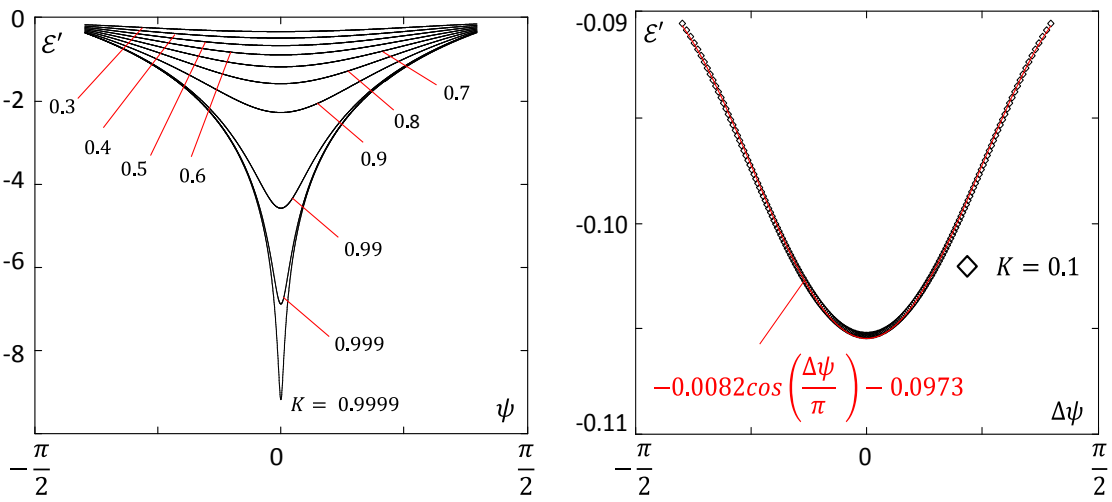

Figure 22. $\mathcal{E}^{\prime}(\psi)$ for $K=0.3 \div 1$ (left) and $K=0.1$ (right).

To compare shapes of $\mathcal{E}^{\prime}(\psi)$ and $\mathcal{E}(B)$ wells, we need to bring their arguments to the same scale. We do this with a help of normalized distance to the attractor $\beta$, which in this case we define as

$$
\beta=\frac{\psi}{\frac{\pi}{2}} .
$$

Wells $\mathcal{E}(B)$ and $\mathcal{E}^{\prime}(\beta, K=1)$ with reduced arguments are shown side by side in Figure 23. The calculated points (rhombs) fall on red curves describing logarithmic functions $-\ln |\beta|$ and $-2 \ln |\beta|$ respectively. The data suggest that for all $\beta$-values

$$
\mathcal{E}^{\prime}(\beta)=2 \mathcal{E}(\beta)
$$

This equality suggests that the energy (thus the asymptotic stability) of two strongly coupled and synchronized cells is equal to the doubled energy of a single cell, which is in accord with energy extensivity.
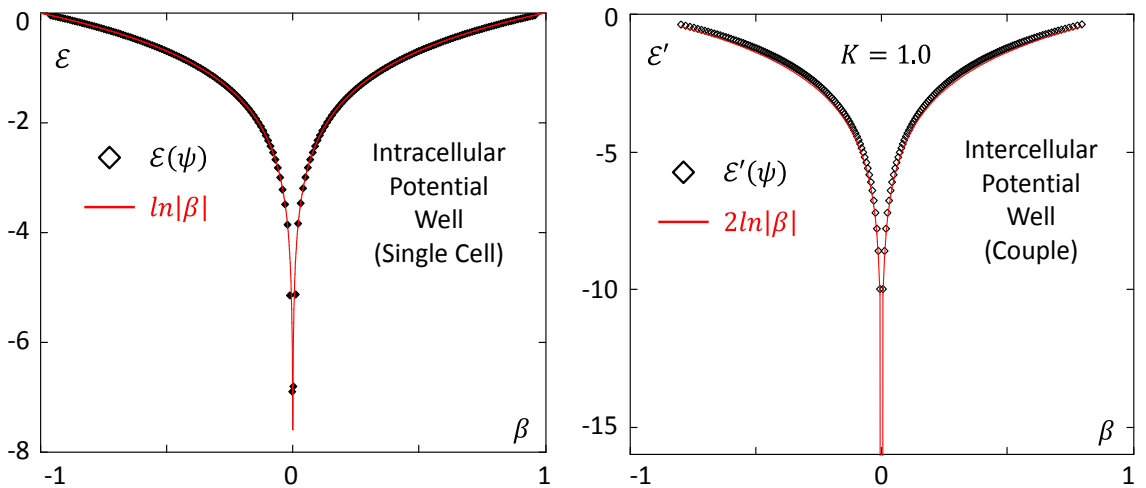

Figure 23. Single cell potential well $\mathcal{E}(\beta)$ (left) and potential well for strongly coupled and synchronized couple $\mathcal{E}^{\prime}(\beta)$ (right).

Synchronization process we considered above describes operation of selfsustained oscillators with periodical waveforms and can be applied to the circular cellular flows. Beside circular, cells possess a periodic radial flows. In fact, both types of flows involve the same dust particles, and being nonlinear, tend to 
synchronize. For example, converging evolution functions $\chi_{n}$ in Figure 3.1 have oscillating components with period $2 t$.

To illustrate how radial flows may synchronize, we can employ Wick's rotation, $t \rightarrow-i t$. This transformation converts real exponents $\exp (\mathcal{E} t)$ of converging evolutions into imaginary exponents $\exp (i \omega t)$ of periodical processes

$$
\exp (\mathcal{E} t) \stackrel{\substack{\omega=-\mathcal{E} \\ t-\text {-it }}}{\Rightarrow} \exp (i \omega t) \text {. }
$$

Here to avoid confusion, we use two different parameters for imaginary time and real time, $\omega=-\mathcal{E}$.

The synchronization schema is shown in Figure 24.

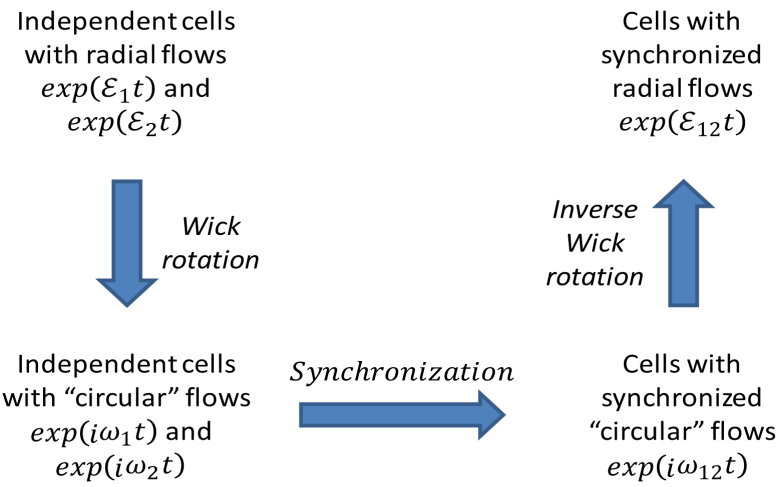

Figure 24. Illustration of synchronization schema under Wick's rotation.

If in the real-time state-space, two cells have close energies $\mathcal{E}_{1} \sim \mathcal{E}_{2}$, in the imaginary-time state-space, they have close frequencies, $\mathcal{E}_{1} \rightarrow \omega_{1}, \mathcal{E}_{2} \rightarrow \omega_{2}$, $\omega_{1} \sim \omega_{2}$. Then they synchronize $\omega_{1} \rightarrow \omega_{12} \leftarrow \omega_{2}$ at frequency $\omega_{12}$, which in the real-time state-space corresponds to the energy $\omega_{12} \rightarrow \mathcal{E}_{12}$. In summary, we obtain that cells synchronize their energies $\mathcal{E}_{1} \rightarrow \mathcal{E}_{12} \leftarrow \mathcal{E}_{2}$.

Not all synchronized cells may have the same frequency. Small amount of them that are at some distance from the superattractors, may synchronize at commensurate frequencies $n \omega_{2}^{\prime}=m \omega_{1}^{\prime}$, where $n$ and $m$ are integers [41]. It is important that even in this case, the cells are subjects to the phase entrainment and their phase differences can be used as network characteristic (order parameter). For simplicity, in the following discussions we assume that all cells synchronize at a single frequency.

A special case is synchronization of spinorial cells. The spinorial cell full period is $2 \pi \mathcal{W}_{j}$ and their phase differences can be as big as

$$
\psi= \begin{cases}2 \pi+\delta \psi & \text { for } Z 2 \text { cells } \\ 6 \pi+\delta \psi & \text { for } Z 3 \text { cells }\end{cases}
$$

where $|\delta \psi|<\pi / 2$ is a small deviation from integer number of $2 \pi$. This happen when the synchronized cells are located at different attractor loops (Figure 25). We define two principally different synchronization modes: in-phase synchronization, $|\psi|=|\delta \psi|<\pi / 2$, and out-of-phase synchronization, $|\psi|=|2 \pi w+\delta \psi|, w=1,2,3$. 

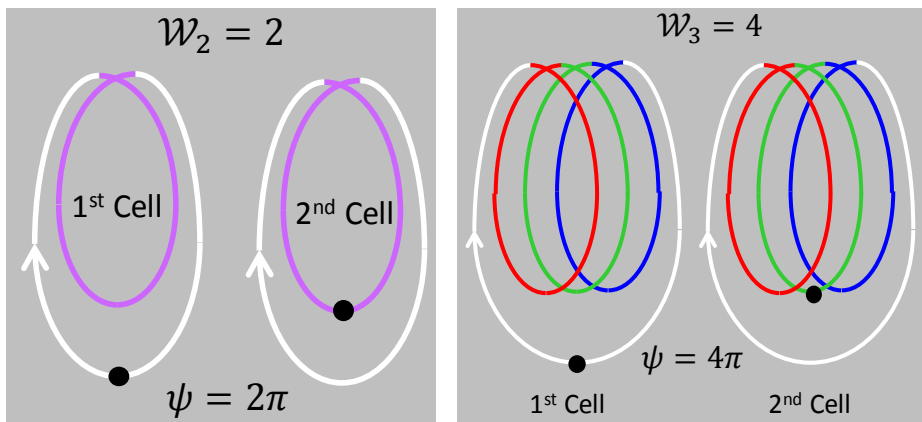

Figure 25. Out-of-phase synchronized d-cells (left) and q-cells (right). Black points show cell locations at their attractors at some time instant.

At normal temperatures, most of the cells are located near the superattractors, operate at close frequencies, and $\delta \psi \rightarrow 0$.

\subsection{Emergence of Global Symmetries. Phase Difference as Order Parameter}

Before being synchronized, cells rotate each with its own pace and count their local time independently. After synchronization, they form coherent networks. They clock time with the same rate. Synchronization is a phase transition that produces a major symmetry: global time scale.

This symmetry provides a basis for unification of scales of other physical parameters. The list includes energy $\mathcal{E}$, temperature $\theta$, asymptotic stability $\lambda$, critical temperature of cell dissociation/formation $\theta_{c}$, and chemical potentials $\mu$, which were defined as derivatives of time intervals.

In coupled cell networks, phase entrainment creates a new order parameterphase difference $\psi$ between the cells. If we zoom out a synchronized cellular network, its granular (cellular) structure smoothes out, and the network acquires features of fiber bundle (Figure 26), where the base space is physical space and the cellular dynamics are elevated to the fibers.

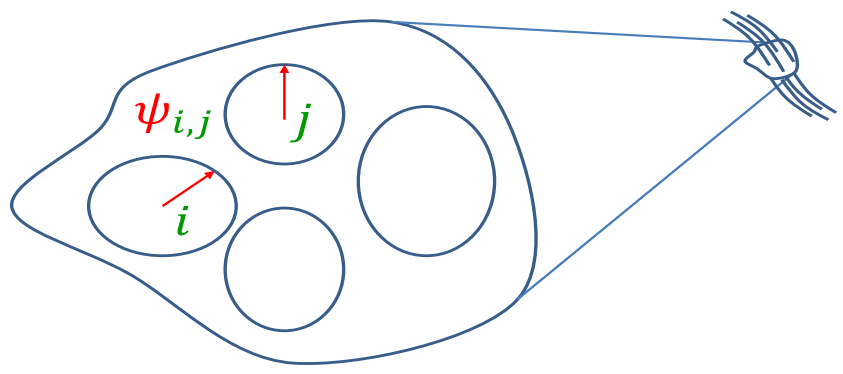

Figure 26. Zoomed-out synchronized cellular network resembles fiber bundle.

Due to the phase entrainment, the fibers are inherently connected, and the local phase difference $\psi$ between cells (now between fibers) acquires function of a field variable.

Each fiber stems from its own cell and inherits the cell topology. Respectively, we can discriminate fibers with simple rotations from the spinorial fibers. Fiber bundle constructions emergent from $Z 1, Z 2$, or $Z 3$ cells, resemble respectively 
electromagnetic, weak, or strong fields of the standard model.

Spinorial cells can form diverse out-of-phase synchronization patterns, and toy particles (as we call them) constructed from the spinorial cells inherit symmetries of their synchronization patterns.

Synchronized cellular networks comprise cells of different geometry and sizes. It does not concern with distances between the cells. The networks are not crystal lattices and they do not provide space gauges. However, it is still possible to calibrate space intervals on a premise that we have had a universal time scale and, in addition, postulate that network excitations propagate through the network with a constant speed $c$. Borrowing this postulate from relativity, we can define a standard space interval $\mathcal{X}$ as

$$
\mathcal{X}=c \mathcal{T},
$$

where $\mathcal{T}$ is the standard time interval (cell rotation period).

This definition obviously connects space and time scales, but it does not establish sameness between space and time. Time remains to be irreversible and space-independent.

$\mathcal{T}$-based time scale is not the only time scale that emerges in cellular networks. Later we will discuss dilated time resembling that of general relativity.

There exists one more symmetry related to our approach that is worth mentioning. The important feature of iteration function (2.6) is that it has one and the only extremum. This feature is called unimodality. Because of this feature, the function belongs to the class of Feigenbaum universality. If one replaces this function with another function from this class, most of the emergent phenomena we encounter in this paper will be preserved. (S)he would find a similar bifurcation diagram, alike probability distributions, the same sets of discrete states, quantized charges, spinorial cells, the same values of chemical potentials, and so on. Even the charge conjugation symmetry can be recovered with a new function if it has a smooth extremum, which can be approximated by a quadratic parabola, by properly selecting coordinate system and its origin.

\subsection{Quantum Distributions}

In Section 3.1, we considered synchronization phenomenon called phase entrainment. Each of coupled cells in the network forces the other cells to change their energies toward its own value. After iterative "negotiations" the cells come to a common trade-off value. If we have more than two cells in the network and some of them have been already synchronized, the synchronized cells have progressive advantage. This nonlinearity creates a positive feedback: the more cells in the network have been synchronized at a given energy, the higher is the probability to get another cell at the same energy. Like in the case with iron filing described above, synchronization spontaneously break the original, almost continuous, cell distribution and create a discrete spectrum (Figure 27). Cell synchronization also alternates the Boltzmann-like cell distribution (2.36) that we encountered in the previous chapters. 


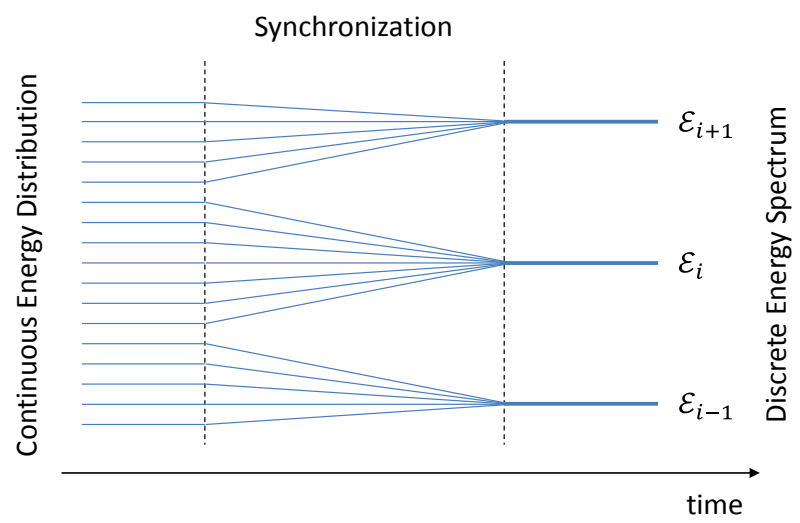

Figure 27. Synchronization alternates cell distribution among the energies.

To formalize the effect of phase entrainment, let us consider a group of cells some of which have been synchronized at states with energy $\mathcal{E}$. Let the rest of the cells in this group have close but different energies. The synchronized cells force the latter to synchronize at the same energy. Let the initial probability to find a cell at state $\mathcal{E}$ is $w$ and the resulting probability is $w_{+}$. In simplest case, the probability to get a new cell at the same state is proportional to the number of cells at this state $\kappa_{+} w_{+}$, where $\kappa_{+}$is the proportionality coefficient. The total number of the new cells is $\kappa_{+} w_{+} w$, and the total number of all cells at state $\mathcal{E}$ after adding the new cells is

$$
w_{+}=w\left(1+\kappa_{+} w_{+}\right),
$$

Signs "+" in (3.11) indicates that the synchronization leads to phase-entrainment (which is not the only possibility).

By solving Equation (3.11) for $w_{+}$, we obtain that

$$
w_{+}=\frac{1}{\frac{1}{w}-\kappa_{+}},
$$

and after substitution of Boltzmann-like factor $w=w_{j}(\mathcal{E}, \theta)$, where $w_{j}(\mathcal{E}, \theta)$ is given by partition function (2.36), in (3.12), we obtain a new partition function

$$
w_{+, j}(\mathcal{E}, \theta)=\frac{\kappa_{+}^{-1}}{\left(\kappa_{+} C_{0}\right)^{-1} \exp \left(\frac{\mathcal{E}+\mu_{j}}{\theta}\right)-1} .
$$

Remarkably, Equation (3.13) has Bose-Einstein distribution form. It is obtained without requirement of the identity of the participating cells!

Phase entrainment is not the only synchronization scenario. At the opposite pole, synchronization may quench oscillations [40] [45] [46] [47] [48], the phenomenon known also as amplitude death or oscillation cessation. Quenching scenario depends on the details of involved oscillators, their coupling mechanisms, and other conditions. Applying to vacuum cells, we assume that quenching destroys cells as self-organized entities and they just dissolve into the dust background. We assume that quenching is also affected by a positive feedback. However, this time the cells compete not with their rivals, but with the noisy background. 
The more cells ceased to exist the stronger is the background forces destroying the remaining cells.

To describe cell distribution under quenching scenario, we use equations similar to (3.11) where we replace probability $w_{+}$with $w_{-}$, coefficient $\kappa_{+}$with $\kappa_{-}$, and sign "+" in parentheses with sign "-" to keep $\kappa_{-}>0$. After these replacements we obtain that

$$
w_{-}=w\left(1-\kappa_{-} w_{-}\right) .
$$

Signs "-" in (3.14) indicates that the synchronization leads to the cellquenching.

By solving (3.14) for $w_{-}$, we obtain that

$$
w_{-}=\frac{1}{\frac{1}{w}+\kappa_{-}},
$$

and after substitution of $w=w_{j}(\mathcal{E}, \theta)$ in (3.15), we obtain that cell distribution for quenching scenario is

$$
w_{-, j}(\mathcal{E}, \theta)=\frac{\kappa_{-}^{-1}}{\left(\kappa_{-} C_{0}\right)^{-1} \exp \left(\frac{\mathcal{E}+\mu_{j}}{\theta}\right)+1} .
$$

Distribution (3.16) has Fermi-Dirac distribution form.

We demonstrated that synchronization may transform the original distribution of independent cells into distributions typical for quantum-mechanical objects. Unlike quantum mechanics, where quantum statistics are intimately connected to the strict identity of particles, dissipative cells synchronize and form quantum distributions even if they are not copies of each other. This is a big relief from the enormous constraint. This is the power of synchronization, the power of asymptotic stability.

\subsection{Synchronized Spinorial Cells as Particle Building Blocks}

In this paper, we consider three types of cells belonging to zones $Z 1, Z 2$ and $Z 3$. Their attractors are shown in Figure 28. Each attractor loop has its own color in the correspondence with the accepted charge-color code shown in Figure 17.

White loops represent quasi-neutral states/phases, purple loop represents $\pm e$-charged phases, and red, green, and blue loops represent color-charged phases. Color-charge phases are ordered. They create a cyclic semi-group. The order for cells (anti-cells) is always the same and unidirectional: $\ldots \rightarrow$ neutral $\rightarrow$ red $\rightarrow$ green $\rightarrow$ blue $\rightarrow$ neutral $\rightarrow \ldots$.

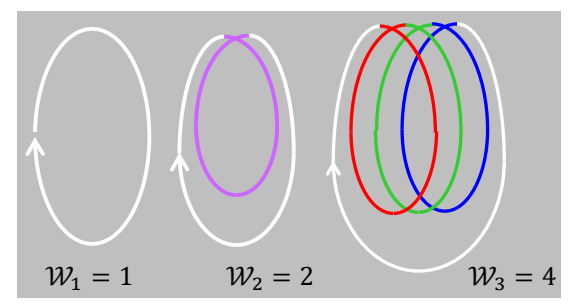

Figure 28. Attractor loop diagrams for $Z 1, Z 2$, and $Z 3$ type cells. 
With a few exceptions, we consider cellular networks comprising mostly of $Z_{1}$-cells having single-loop attractors $\left(\mathcal{W}_{1}=1\right)$. When consider complexes of $Z 2$ and Z3 spinorial cells, which have more complex attractor topology $\left(\mathcal{W}_{2}=2, \mathcal{W}_{3}=4\right)$, we assume that they are immersed in $Z 1$ network as topological defects. Two, or more, directly coupled spinorial cells constitute a toy particle.

Each couple of synchronized cells carries a connecting link, that represent a special interest. We associate the connecting links with mediators of the corresponding fields. In case of synchronized spinorial cells, the connecting links inherit spinorial features from their host-cells and we call them spinorial links. Unlike regular links that occur between connected $Z 1$-cells, the spinorial links are localized. They cannot travel across the network without their host-cells. Like extremely heavy W-bosons and confinement gluons, the spinorial links are doomed to be internal particle features.

To synthesize a toy particle, we are equipped with only four types of building blocks: Z2 spinorial cells and anti-cells, and Z3 spinorial cells and anti-cells. Their combinations provide six different link types. This number is multiplied by the number of different spinorial-cell synchronization patterns (Figure 25). Different links may have different coupling strengths and carry different energies, that should diversify particle masses. However, these particle features are out of the scope of this paper.

Examples of spinorial links are illustrated in Figures 29(a)-(c). Not all of the possible spinorial links have their analogs in the standard model. For example, a link between a neutral spinorial cell and a charged spinorial cell shown in Figure 29(d), or a link between d-cell and q-cell.
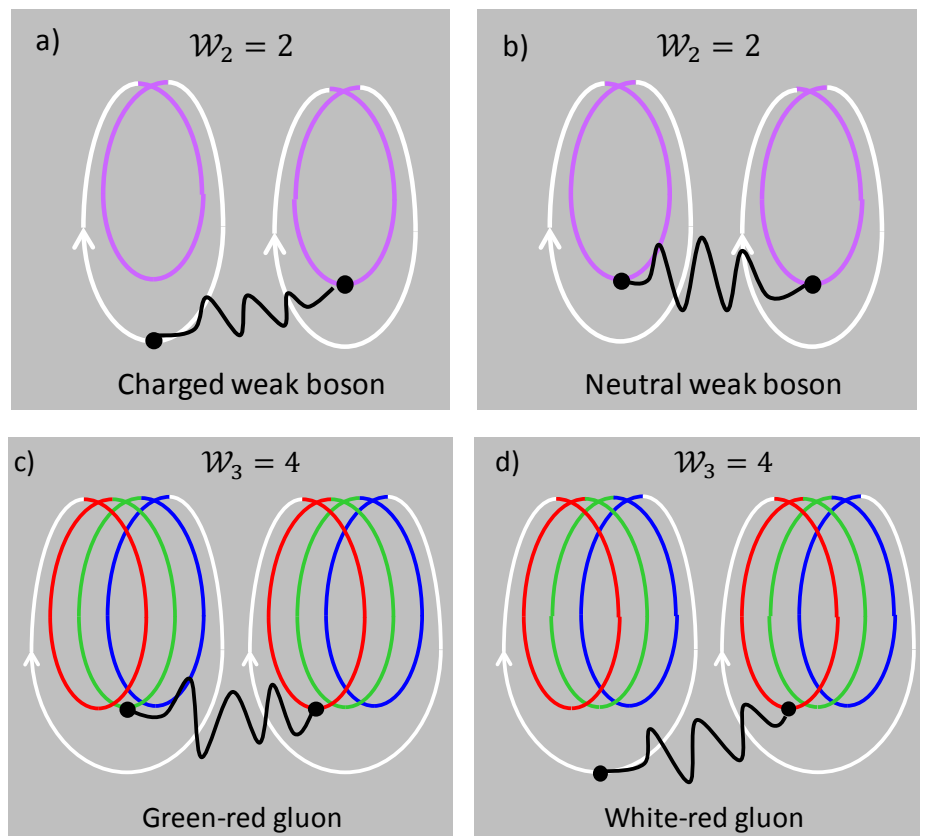

Figure 29. Spinorial cell links as localized bosons (shown by wavy lines): (a) charged weak boson; (b) neutral weak boson; (c) green-red gluon (d) white-red gluon. 
Despite that the synchronized cell networks not necessary create a lattice, it is tempting to draw a parallel between the cell connecting links and solid-state phonons. In this respect, we would associate links between the in-phase synchronized cells with acoustic phonons, and links between the out-of-phase synchronized spinorial cells as optical phonons. Like their phonon counterparts, we expect that the "acoustic" links and "optical" links have different dispersions and different masses.

While building the toy-particles from the spinorial cells, we avoid in-phase synchronization between directly connected spinorial cells (light "acoustic" links) assuming that they are not stable enough.

Out-of-phase spinorial links carry at least one charge. Their charges rotate in synchrony with their host cells. Here is an example of "gluon"-link charge rotation in time

$$
\begin{gathered}
\text { 1st cell } \cdots \rightarrow \text { neutral } \rightarrow \text { red } \rightarrow \text { green } \rightarrow \text { blue } \rightarrow \text { neutral } \rightarrow \cdots \\
\text { 2nd cell } \cdots \rightarrow \text { red } \rightarrow \text { green } \rightarrow \text { blue } \rightarrow \text { neutral } \rightarrow \text { red } \rightarrow \cdots \\
\text { Gluon } \cdots \rightarrow\left(\begin{array}{c}
\text { neutral } \\
\text { red }
\end{array}\right) \rightarrow\left(\begin{array}{c}
\text { red } \\
\text { green }
\end{array}\right) \rightarrow\left(\begin{array}{c}
\text { green } \\
\text { blue }
\end{array}\right) \rightarrow\left(\begin{array}{c}
\text { blue } \\
\text { neutral }
\end{array}\right) \rightarrow\left(\begin{array}{c}
\text { neutral } \\
\text { red }
\end{array}\right) \rightarrow \cdots
\end{gathered}
$$

To avoid long phrases, we provide nicknames to the spinorial cells. They are listed in Table 3.

Table 3. Nicknames for spinorial cells.

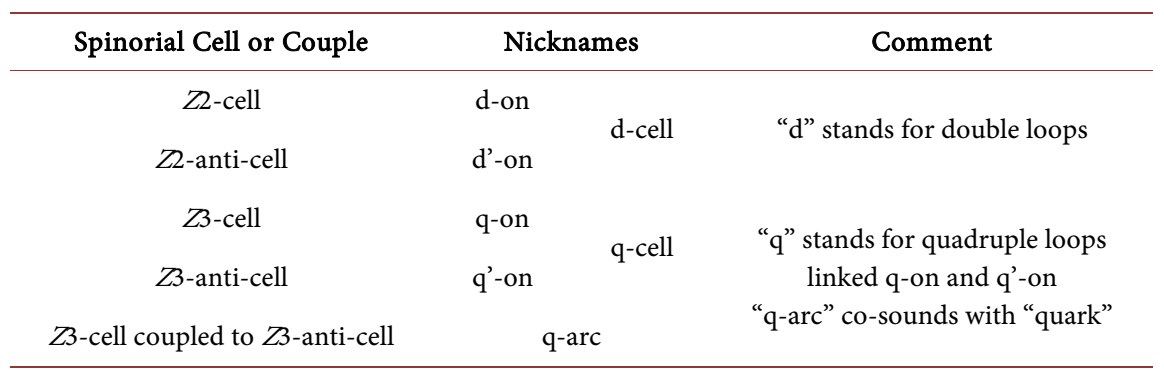

In the paper, we use a number of illustrations/diagrams to better communicate the ideas. In the following discussions we will use one more type of diagrams, circular time-diagrams that linked to the other type diagrams as it is shown in Figure 30.

Upper line diagrams illustrates position on the bifurcation diagram (Figure 30(a)) and temporal behavior of $Z 2$-cells: waveform (Figure 30(b)), loop-diagram (Figure 30(d)), and circular-time diagram (Figure 30(c)).

Bottom line diagrams illustrate $Z 3$-cell dynamics.

Each circular time-diagram represents one full period $\mathcal{T}$ of a single cell. Attractor loops are represented by colored sectors according to the used above charge-color code. The upper sector (twelve o'clock position) shows current charge (state). The diagrams rotate in time, always counterclockwise. The cell rims are black in contrast to the anti-cell rims, which are red. In addition, d-on and d'-on diagrams expose the cell charge polarity marked by “-” and "+” signs. 

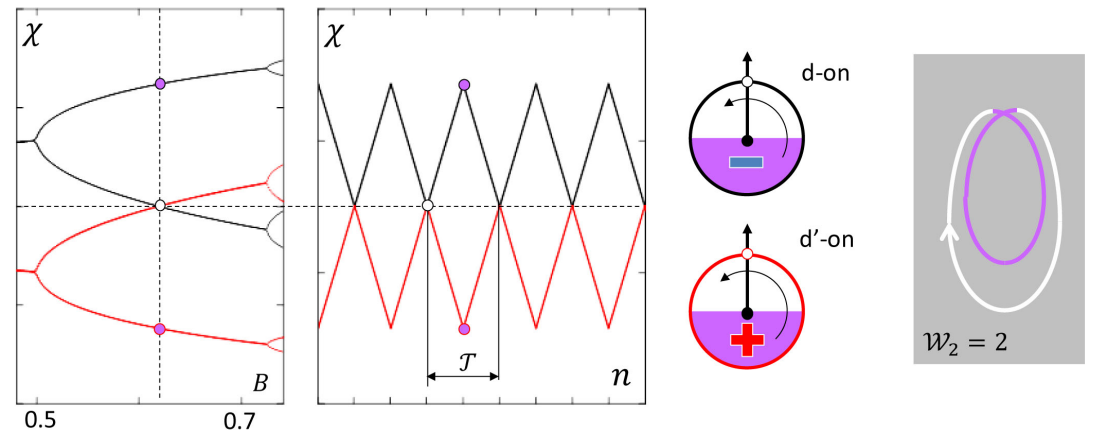

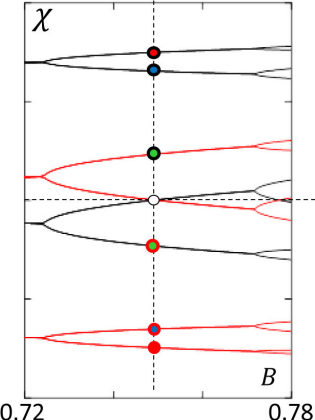

(a)

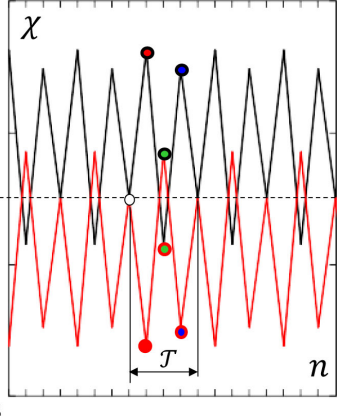

(b)
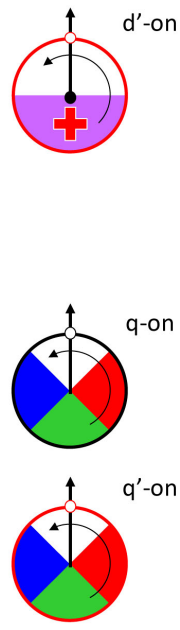

(c)

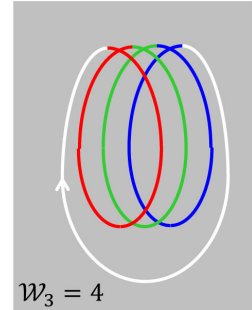

(d)

Figure 30. (a) Bifurcation diagrams; (b) Charge trajectories; (c) Circular time diagrams; d: Attractor loops.

Examples of circular time diagrams of in-phase and out-of-phase synchronized spinorial couples are shown in Figure 31. The formers have alike sectors at identical positions.
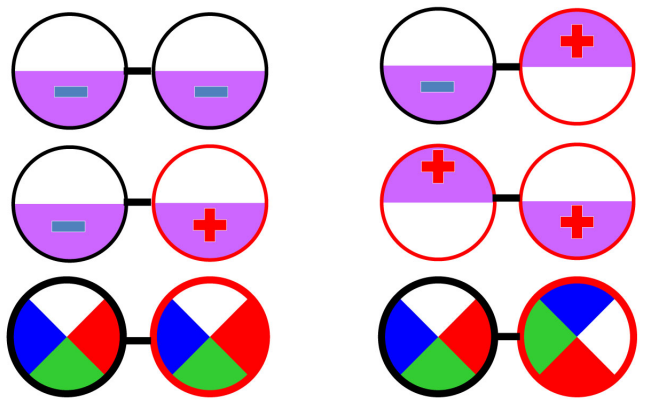

Figure 31. Examples of in-phase synchronized couples (left) and out-of-phase synchronized couples (right).

\section{When building the toy particles, we use the following rules:}

- the stable spinorial links are links between out-of-phase synchronized couples;

- $\mathrm{d}$-cells/anti-cells are the only carriers of the electric charge $( \pm e)$;

- d-cells/anti-cells are also the only carriers of $\left(\begin{array}{c}u p^{+} \\ \text {down }\end{array}\right)$-and $\left(\begin{array}{c}u p^{-} \\ d o w n\end{array}\right)$-flavors;

- $\mathrm{q}$-arcs are the only carriers of color charges and $\left(\begin{array}{llll}u / d & c / s & x / y & t / b\end{array}\right)$ -flavors. They are electrically neutral;

- in-phase synchronized q-arc is a carrier of unstable $x / y$-flavor and represents 
an intermediate (unstable) state;

- toy leptons consist of coupled d-cells;

- toy hadrons consists of coupled d-cells and q-arcs.

Flavor assignments illustrated by circular time diagrams are shown in Figure 32.

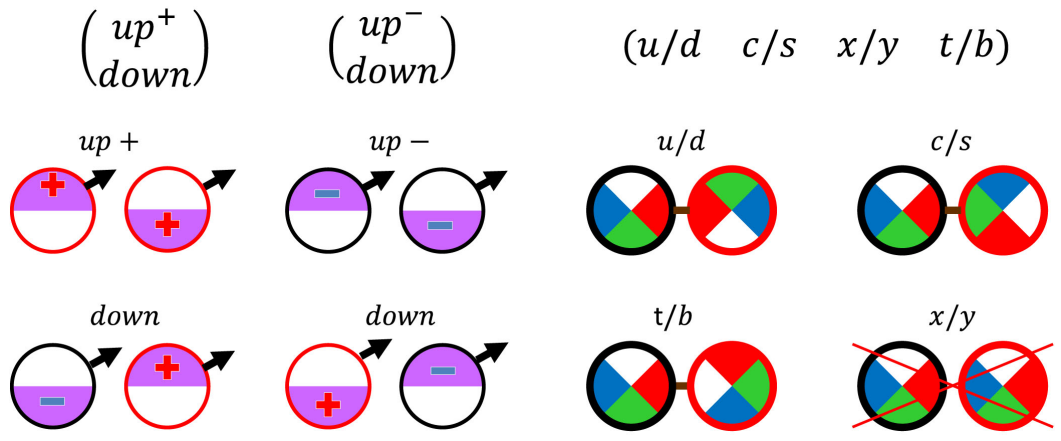

Figure 32. Flavor assignment diagram. Small arrows attached to $d$-cells represent links to q-arcs.

To match the set of quark flavors of the standard model, we mix q-cells/anticells carrying $\left(\begin{array}{lll}u / d & c / s & t / b\end{array}\right)$-flavors and d-cells/anti-cells carrying $\left(\begin{array}{c}u p^{ \pm} \\ \text {down }\end{array}\right)$ -flavors and obtain the desired flavor set as direct (Cartesian) product

$$
\left(\begin{array}{lll}
u & c & t \\
d & s & b
\end{array}\right)=\left(\begin{array}{lll}
u / d & c / s & t / b
\end{array}\right) \times\left(\begin{array}{c}
u p^{ \pm} \\
\text {down }
\end{array}\right)
$$

We avoid fractional electric charges by representing hadrons as toy lepton/q-arc mixtures.

Full set of q-arc flavors $\left(\begin{array}{llll}u / d & c / s & x / y & t / b\end{array}\right)$ with $x / y$ placed properly between $c / s$ and $t / b$ forms a cyclic semi-group (Figure 33). Group generator $g$ produces unidirectional $\pi / 2$-rotation of one of the two coupled circular time diagrams. Each $\pi / 2$-diagram-rotation corresponds to cell transition from one attractor loop to the next one, or $2 \pi$-phase shift in the cell dynamics. The reverse rotations are prohibited because of time irreversibility. Instead, we rotate the complimentary diagram in the same direction. We use this symmetry (or anti-symmetry) when discussing flavor mixing matrix.
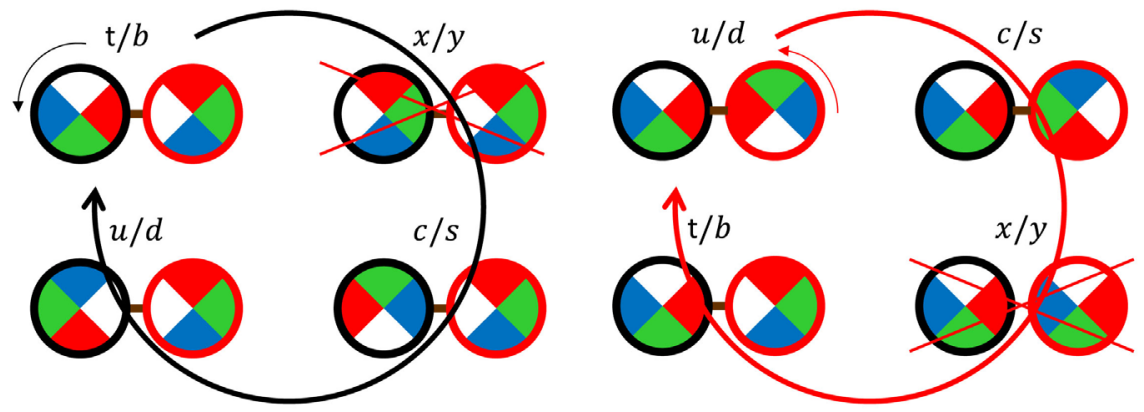

Figure 33. Q-arc flavors $\left(\begin{array}{llll}u / d & c / s & x / y & t / b\end{array}\right)$ form a cyclic semi-group. 
Beside different compositions and synchronization patterns, toy cells may possess different stereometry, architectures, and spinorial links. For example, cell permutations inside a particle may create a new particle(s).

Anti-particles are obtained from the corresponding particles by replacing all cells with their inversed counterparts, while preserving the original geometry and synchronization patterns.

\section{Toy Particles}

We associate small groups of synchronized and directly linked spinorial cells with toy particles. Toy particles are immersed into $Z 1$-cell "electromagnetic" network. There exist four types of the toy particle building blocks: d-on, d'-on, q-on, and q'-on. The diversity of toy particles comes not only from different compositions but also because of their diverse synchronization patterns and architectures. Below, we consider a few examples of toy particles. They do not exhaust all possibilities but rather illustrate the power and capabilities of cellular dynamical networks.

\subsection{Toy Leptons Spin}

We build toy leptons exclusively from d-cells/anti-cells. Each directly coupled pair is synchronized out-of-phase. One possible arrangement of toy-leptons is shown by their circular time diagrams in Figure 34.
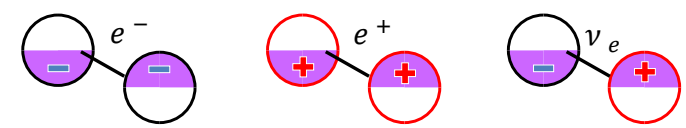

$1^{\text {st }}$ Generation
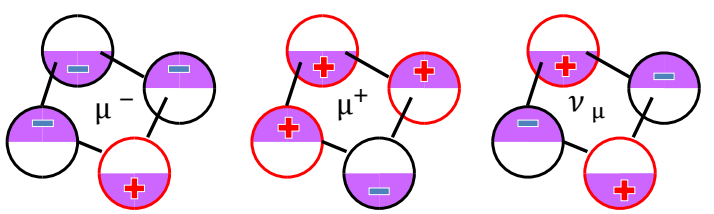

$2^{\text {nd }}$ Generation
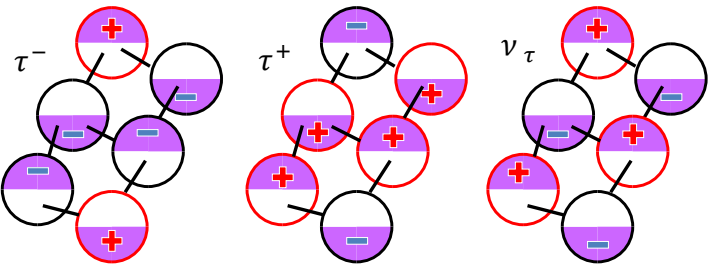

\section{$3^{\text {rd }}$ Generation}

Figure 34. Toy lepton family. Electric charge pattern mimics pattern of real particles.

The charge pattern of toy leptons mimics the charge pattern of the standard model leptons. Like real leptons, the toy lepton family consists of three generations. Each generation has one positively charged particle, one negatively charged particle and one $\left(v_{e}\right.$ and $\left.v_{\mu}\right)$ or two $\left(v_{\tau}\right)$ neutral particles (in Figure 34 only one $\tau$-neutrino is shown).

Toy electron $e^{-}$consists of two out-of-phase synchronized d-ons (Figure 34 and Figure 35)). When one cell of the couple is in the charged phase, the other cell is electrically neutral, and vice versa. These charge oscillations create im- 
pression that the charge is moving in space from one cell to the other and backward (see Figures 35). However, the charge "motions" in space are not real, and their apparent "speed" may exceed the speed of light without confronting the relativity principles. The surrounding cells response to the charge pulsations by inducting currents $i$. Currents cannot go directly through $\mathrm{d}$-ons. Therefore, the currents spontaneously break symmetry and create current loops. The current directions and orientation are established randomly through their recurrent interactions with $\mathrm{d}$-ons. Their stability is fragile and their patterns can be easy alternated by perturbations. However, at normal conditions, the current producing oscillating charge $(-e)$ is rather a constant. The cell oscillation period $(\mathcal{T})$ is also a constant. Therefore, the induced current loops and the magnetic moments they create are also constants. We associate the phenomenon with the toy particle spin. It is obviously due to the spinorial features of d-ons (two-loop attractors) and out-of-phase synchronization.

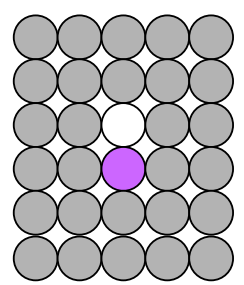

$t$

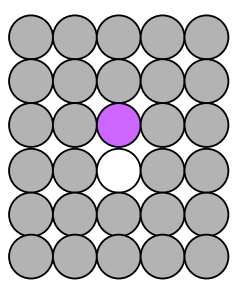

$$
t+\mathcal{T} / 2
$$

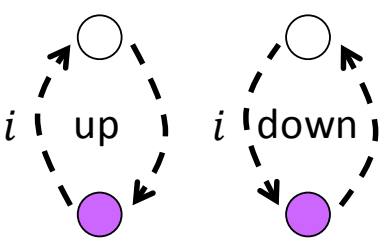

Figure 35. Diagram explaining origin of toy particle spin. Pulsating charges of out-ofphase synchronized d-ons (two left diagrams) induct current loops and magnetic moments in the surrounding network (two right diagrams). Purple and white circles represent d-ons in charged and neutral states respectively. Grey circles represent surrounding electromagnetic cells.

Returning to Figure 28, toy positron, $e^{+}$, consists of two out-of-phase synchronized d'-ons. A neutral couple of d-on and d'-on plays the role of the electron neutrino, $v_{e}$.

The second generation $\left(\mu^{-}, \mu^{+}\right.$and $\left.v_{\mu}\right)$ is constructed by adding one d-on and one d'-on to each particle of the first generation. The additions do not change the charge pattern, but rather increase the particle energy (mass) and, most likely, affects its stability (lifetime).

The third generation $\left(\tau^{-}, \tau^{+}\right.$and $\left.v_{\tau}\right)$ is obtained the same way as the second generation, by adding two more complimentary charged d-cells to each particle of the second generation. Unlike two previous generations, the approach allows to obtain two different tau-neutrinos, $v_{\tau}$ (only one is shown in Figure 34).

\subsection{Toy Hadrons}

We assemble toy hadrons as combinations of q-arcs and d-cells/anti-cells. Qarcs provide $(u / d \quad c / s \quad t / b)$-flavors to hadrons, and d-cells/anti-cells provide $\left(\begin{array}{c}u p^{ \pm} \\ \text {down }\end{array}\right)$-flavors and electric charges. Direct product of $\left(\begin{array}{llll}u / d & c / s & t / b\end{array}\right)$ 
-flavors and $\left(\begin{array}{c}u p^{ \pm} \\ \text {down }\end{array}\right)$-flavors provides a set of six flavors resembling those of the standard model quark flavors.

Toy mesons consist of one $\mathrm{q}$-arc couple and two d-cells/anti-cells. Coupled circular time-diagrams of a group of toy mesons comprising $u / d$-flavored q-arcs are shown in the left column in Figure 36. The group consists of one positively charged particle carrying flavor $u^{+}=(u / d) \times\left(u p^{+}\right)$, one negatively charged particle carrying flavor $u^{-}=(u / d) \times\left(u p^{-}\right)$, and two neutral particles carrying two different flavors $d=(u / d) \times($ down $)$. The latter differ by their architectures: in one of them, d-on is connected to q'-on and d'-on is connected to q-on, in the other, $\mathrm{d}$-on is connected to q-on and d'-on is connected to q'-on.

A similar meson quartet comprising $c / s$-flavored q-arcs is shown in the right column in Figure 36.

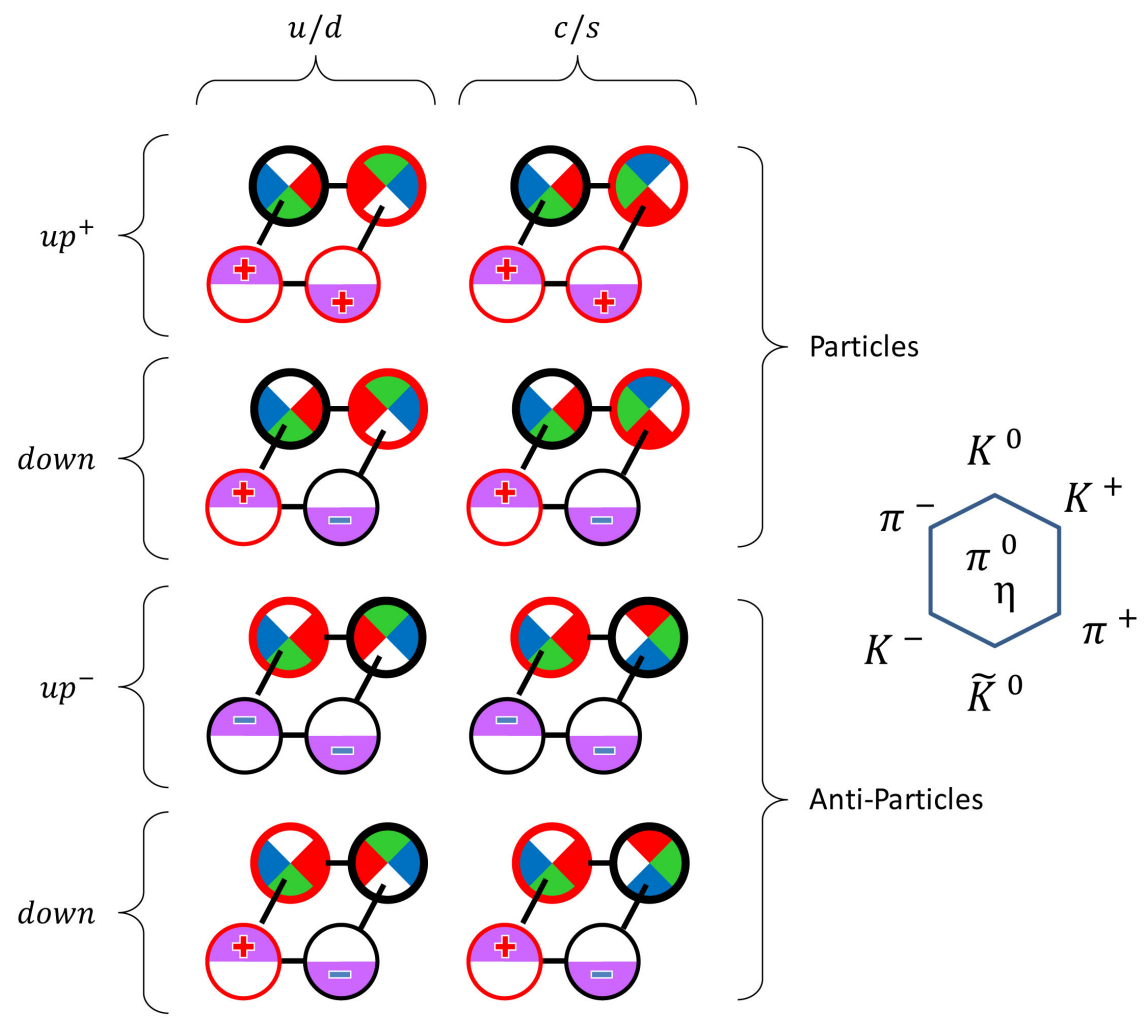

Figure 36. Quartets of $u / d$ - and $c / s$-flavored toy mesons have the same electric charge patterns as quartets of real $K$ - and $\pi / \eta$-mesons that constitute eight-way octet shown in the diagram at right.

The two toy-meson quartets have the same charge pattern as octet of real particles shown in "eight-way" diagram at the right.

In the same way we can assemble a meson quartet carrying $t / b$-flavored q-arcs.

We can significantly extend the toy meson family by adding other geometries, like "stars", "open chains", and "tetrahedrons" shown in Figure 37. 

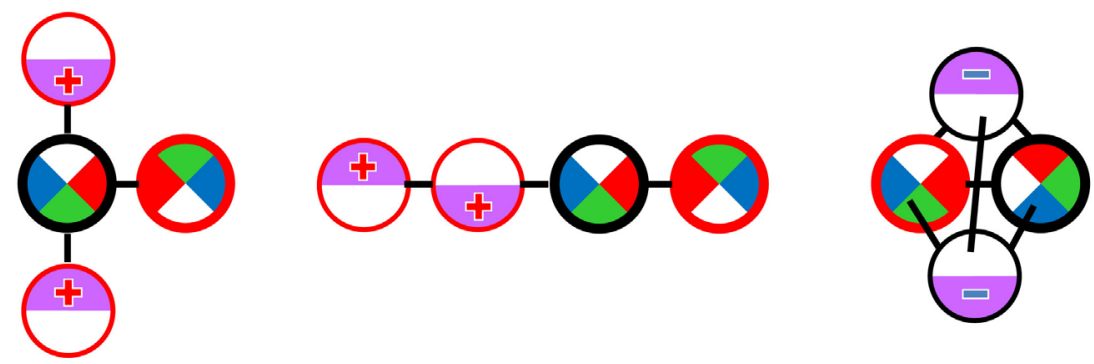

Figure 37. Different toy mesons as spinorial cell "molecules".

By adding one more q-arc to a toy meson we obtain a toy baryon. The baryons carry the same charge patterns but the number of different flavor combinations is significantly increased. Baryon flavors are direct products of

$$
\left(\begin{array}{llll}
u / d & c / s & t / b
\end{array}\right) \times\left(\begin{array}{lll}
u / d & c / s & t / b
\end{array}\right) \times\left(\begin{array}{c}
u p^{ \pm} \\
\text {down }
\end{array}\right)
$$

In Figure 38 we show an example of a toy baryon octet based on $u / d$ - and $c / s$-flavored q-arcs. The baryons have octahedron geometry. To prevent the diagram obstruction, links between d-ons and q-ons are not shown. The octet consists of two positively charged particles, two negatively charged particles, and four neutral particles. The toy baryon octet has a charge pattern similar to that of the real baryon octet comprising $u, d$ and $s$ quarks (Figure 38, the diagram at right). Two $u / d$-flavored particles (left column) are associated with proton and neutron.

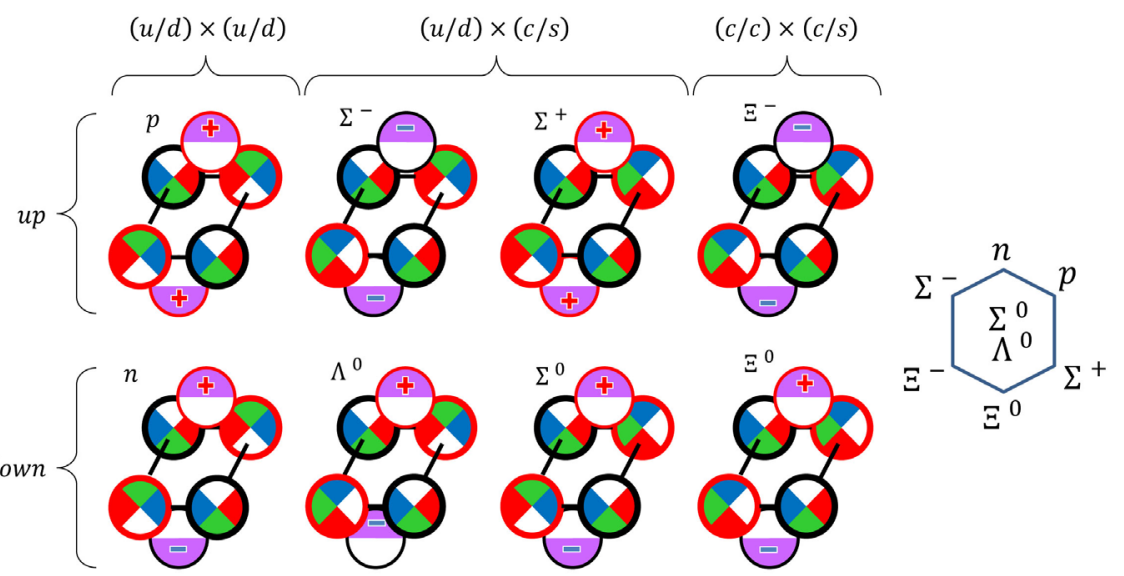

Figure 38. Octet of $u / d$ - and $c / s$-flavored toy baryons has the same electric charge pattern as octet of real baryons consisting of $u$-, $d$-, and $s$-quarks ("eight-way" diagram at right).

An example of "chemical reaction" between toy particles is shown in Figure 39. It describes exchange of $\mathrm{d}$-on and d'-on between toy baryon and toy lepton accompanied by $2 \pi$-phase shift. In reality, neutrino should be replaced with anti-neutrino and moved to the right part of the equation. We write it in the presented form to simplify the picture. 


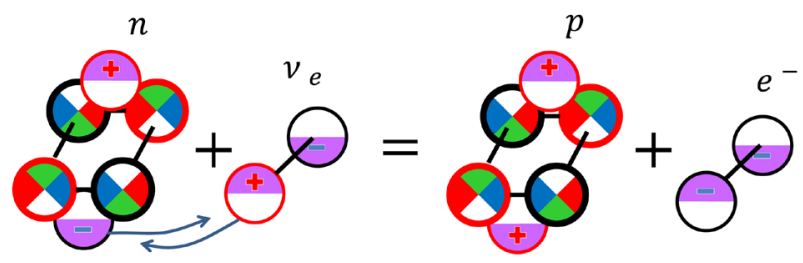

Figure 39. Example of toy particle reaction mimicking neutron disintegration: $n+v_{e}=p^{+}+e^{-}$.

\subsection{Q-Arc Quartet and Flavor Mixing Matrix}

The conventional six quark flavors constitute $3 \times 3$ CKM flavor mixing matrix, which we briefly discussed in Section 1.3. We have observed that the matrix elements $V_{i j}$ can be approximated by the powers of the Feigenbaum delta $\delta_{F}^{-p}, p=0,1,2,3$. The approximate CKM'-matrix is shown in Figure 40(left). We also found that $3 \times 3 C K M^{\prime}$-matrix can be expanded to more symmetric $4 \times 4$ matrix, which is shown in Figure 40(right).

\begin{tabular}{|c|c|c|c|c|}
\hline & & \multicolumn{3}{|c|}{ Down-Type Quarks } \\
\hline & & $d$ & $s$ & $b$ \\
\hline & $u$ & 1 & $\delta_{F}{ }^{-1}$ & $\delta_{F}{ }^{-3}$ \\
\hline & $c$ & $\delta_{F}{ }^{-1}$ & 1 & $\delta_{F}^{-2}$ \\
\hline & $t$ & $\delta_{F}{ }^{-3}$ & $\delta_{F}{ }^{-2}$ & 1 \\
\hline
\end{tabular}

\begin{tabular}{|c|c|c|c|c|}
\cline { 2 - 4 } \multicolumn{1}{c|}{} & $u / d$ & $c / s$ & $x / y$ & $t / b$ \\
\hline$u / d$ & 1 & $\delta_{F}^{-1}$ & $\delta_{F}^{-2}$ & $\delta_{F}^{-3}$ \\
\hline$c / s$ & $\delta_{F}^{-1}$ & 1 & $\delta_{F}^{-3}$ & $\delta_{F}^{-2}$ \\
\hline$x / y$ & $\delta_{F}^{-2}$ & $\delta_{F}^{-3}$ & 1 & $\delta_{F}^{-1}$ \\
\hline$t / b$ & $\delta_{F}^{-3}$ & $\delta_{F}^{-2}$ & $\delta_{F}^{-1}$ & 1 \\
\hline
\end{tabular}

Figure 40. Approximate $3 \times 3 C K M^{\prime}$-quark mixing matrix (left) and $4 \times 4$-matrix q-arc mixing matrix that includes $x / y$ flavor corresponding to in-phase synchronized q-arcs.

Q-cells in q-arcs can synchronize in four different patterns. We associate these patterns with four $\mathrm{q}$-arc flavors $\left(\begin{array}{llll}u / d & c / s & x / y & t / b\end{array}\right)$. They are shown in the $4 \times 4$-matrix in the upper line and left column in the proper order. The matrix elements related to the "unstable" in-phase synchronized q-cells and carrying $x / y$ flavor are shown in pink. Remarkably, the pink cells accommodate all the Feigenbaum delta powers missing in $3 \times 3$ matrix. Moreover, $4 \times 4$ matrix elements are ordered! Their orders are shown by black and red arrows in Figure 41.

\begin{tabular}{|c|c|c|c|c|}
\hline & $u / d$ & $\mathrm{c} / \mathrm{s}$ & $x / y$ & $t / b$ \\
\hline$u / d$ & 1 & $\delta_{F}{ }^{-1}$ & $\delta_{F}{ }^{-2}$ & $\delta_{F}^{-3}$ \\
\hline$c / s$ & $\delta_{F}^{-1}$ & 1 & $\delta_{F}{ }^{-3}$ & $\delta_{F}{ }^{-2}$ \\
\hline$x / y$ & $\delta_{F}^{-2}$ & $\delta_{F}^{-3}$ & 1 & $\delta_{F}^{-1}$ \\
\hline$t / b$ & $\delta_{F}^{-3}$ & $\delta_{F}{ }^{-2}$ & $\delta_{F}^{-1}$ & 1 \\
\hline
\end{tabular}

\begin{tabular}{|c|c|c|c|c|}
\hline & $u / d$ & $\mathrm{c} / \mathrm{s}$ & $x / y$ & $t / b$ \\
\hline$u / d$ & 1 & $\hat{\delta}_{F}{ }^{-1}$ & $\delta_{F}^{-2}$ & $\hat{\phi}_{F}{ }^{-3}$ \\
\hline$c / s$ & $\delta_{F}^{-1}$ & - 1 & $\delta_{F}{ }^{-3}$ & $\delta_{F}^{-2}$ \\
\hline$x / y$ & $\delta_{F}^{-2}$ & $\oint_{F}{ }^{-3}$ & 91 & $\oint_{F}^{-1}$ \\
\hline$t / b$ & $\oint_{F}{ }^{-3}$ & $\delta_{F}^{-2}$ & $\oint_{F}^{-1}$ & - 1 \\
\hline
\end{tabular}

Figure 41. Matrix elements represent an ordered set of Feigenbaum delta powers. 
Each arrow starts and ends at the same diagonal element. Moving from one matrix element to another along the arrow is accompanied by $\delta_{F}$-times reduction of the corresponding mixing amplitude $V_{i j}$. According to q-arc synchronization schema, each step along the arrow corresponds to $2 \pi$-rotation in q-cell dynamics, or in the other words, to a jump from one attractor loop to the next one (see Figure 42 (central diagram)) or one-sector rotation of one of the two coupled circular diagrams (Figure 42(left and right diagrams)).

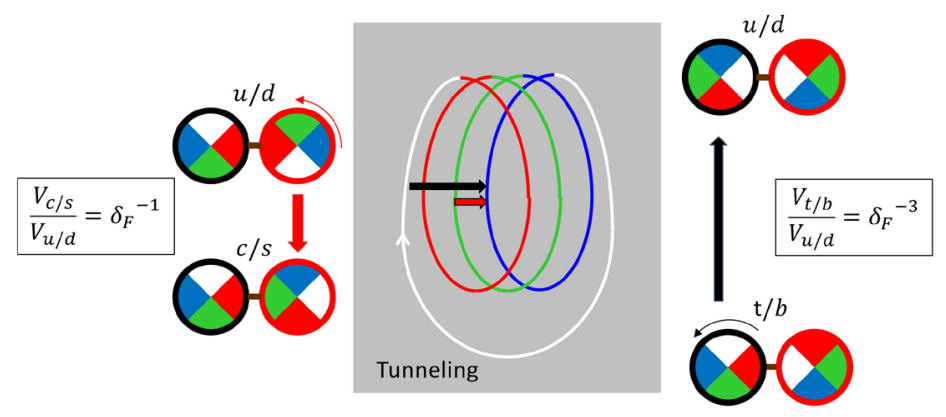

Figure 42. Examples of one-step shift along the red arrow (left) and three-step shift along the black arrow (right), and the corresponding jumps between the attractor loops (center).

All q-arc synchronization patterns and flavor changes covered by $4 \times 4$ matrix are shown in Figure 43 by means of coupled circular time diagrams.

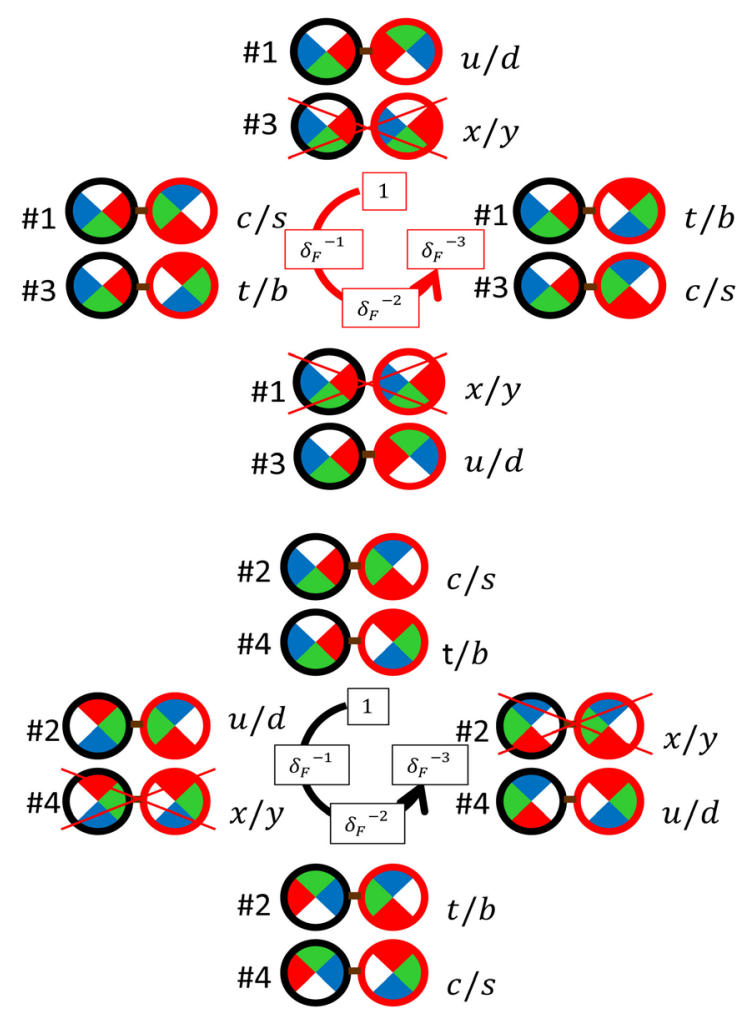

Figure 43. $4 \times 4$ q-arc flavor changing matrix represented by coupled circular time-diagrams. Each diagram couple is marked by the corresponding row/column number and the flavor name. 
Moving farther, we describe transitions from one attractor loop to another (Figures 42(center)) as "tunnel" jumps (Figure 44) between the two synchronization states. The specificity of this "tunneling" is that it occurs not in the real space, but rather in the state-space. We can also say that this is tunneling in time.
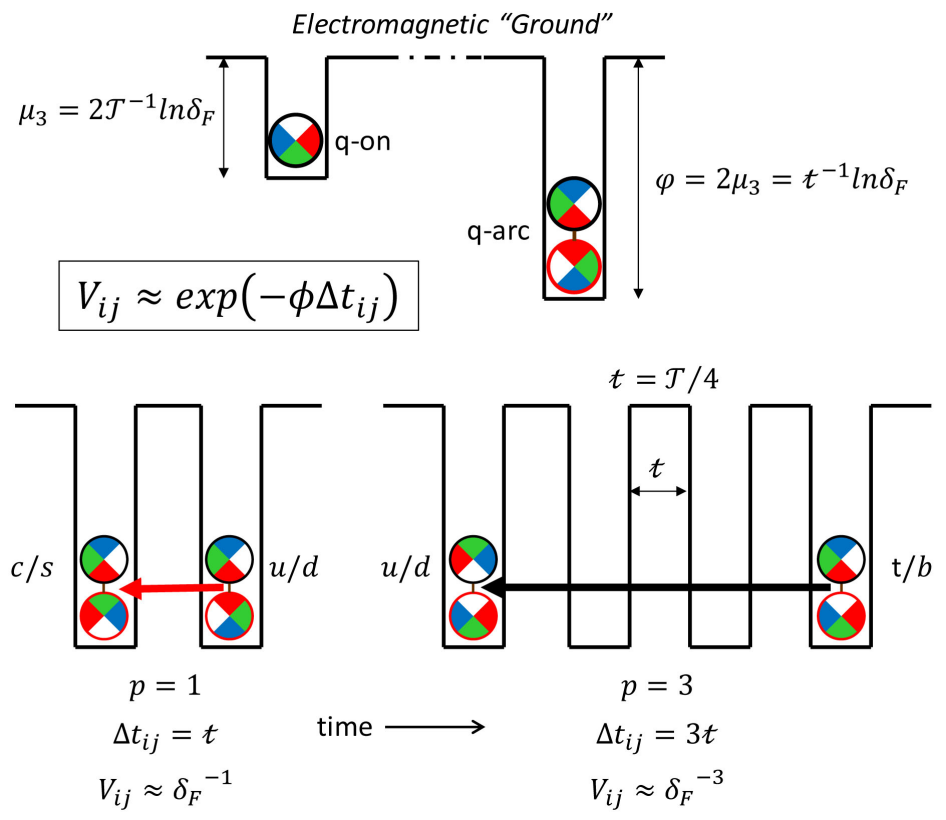

Figure 44. "Tunneling in time". Diagram describes the same processes as shown in Figure 42.

Using the analogy with the space tunneling, we estimate the probability $W_{i j}$ of loop-to-loop "tunnel" jumps. We replace tunnel distances with temporal intervals

$$
\Delta t_{i j}=p t, \quad p=0,1,2,3,
$$

where $t$ is time required for a cell to complete a single loop rotation, which is also the iteration time interval, $p$ is the number of steps along the arrows in Figure 41 diagram and also power in $\delta_{F}^{-p}$.

We assume that the tunnel barrier height $\phi$ is equal to the q-arc internal energy $\mathcal{E}_{q}$, which is approximately doubled energy of a single q-cell or $2 \mu_{3}$ $\left(\mu_{3}=\frac{2}{\mathcal{T}} \ln \delta_{F}\right.$ is chemical potential in zone $\left.Z 3\right)$

$$
\phi=\mathcal{E}_{q} \approx 2 \mu_{3}=2 \frac{2}{\mathcal{T}} \ln \delta_{F}=\frac{1}{t} \ln \delta_{F},
$$

where $\mathcal{T}=4 t$ is q-cell rotation period.

Now, we use the tunnel exponent to estimate the loop-to-loop jump probability which is

$$
W_{i j} \approx \exp \left(-\phi \Delta t_{i j}\right)
$$

After substitution of (4.1) and (4.2) into (4.3), we obtain that probabilities 
$W_{i j}$ are equal to the matrix elements $V_{i j}$

$$
W_{i j} \approx \exp \left(-\frac{p t}{t} \ln \delta_{F}\right)=\delta_{F}^{-p} \approx V_{i j} .
$$

Like all the phenomena we explore in this paper, q-arc flavor transformations are time irreversible. The direction of q-cell rotation is fixed and cannot be reversed, including the direction of the tunnel jumps. If we let cells to tunnel in both directions, the small forward three-step forward jumps probabilities, $\delta_{F}^{-3}$, would be masked by much bigger one-step backward probabilities $\delta_{F}^{-1}$ and the mixing matrix elements would have different values and the matrix itself would have quite different appearance.

\section{4. "Dark Matter"}

The list of electrically neutral toy particles we discussed in previous sections can be extended by adding other toy particles. Some examples are shown in Figure 45 . The picture shows free q-arcs and based on them bigger complexes, and weakly and strongly interacting large cellular clusters. Being electrically neutral, they barely interact with electromagnetic field but carry energy (mass) and together with the previously considered neutral toy particles, can be associated with weakly and strongly interacting "dark" matter.

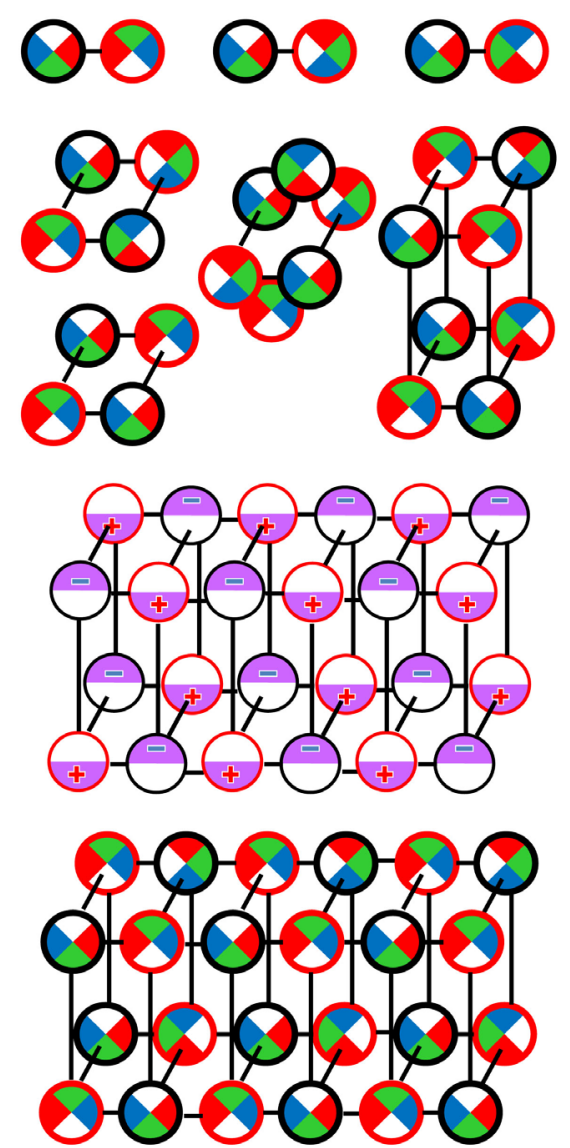

Figure 45. Examples of strongly and weakly interacting electrically neutral spinorial-cell assemblies. 


\section{5. "Relativistic" Phenomena and the Dark Zone}

\subsection{Time Dilation and Related Topics}

We have seen that cell rotation period $\mathcal{T}$ can be used as a natural time unit in synchronized cellular networks. However, we did not provide any clue how to use it in practice. The problem is that with the existing technology and arsenal of instruments, we cannot trace the dust particle trajectories and measure time intervals linked to $\mathcal{T}$. Any imaginary clock would consist of at least two cells, and the clock rate would depend on how quickly the cells can establish their mutual dynamics. Normally, the speed of intercellular interactions is slower than intracellular relaxation, and is parametrized by parameters $\lambda,|\beta|$, or energy $\mathcal{E}$. It is more convenient to have a measure of cell relaxation rate expressed as time intervals. We define cellular relaxation time $\tau$ as

$$
\tau=-\mathcal{E}^{-1} \text {. }
$$

We assume that the clock rate is limited by the cellular relaxation time, and use $\tau$ as a time unit for a "practical" scale.

Unlike the cell rotation period $\mathcal{T}$, relaxation time $\tau$ is not network invariant. Different network regions may have different energies thus different $\tau$-scale based clock rates. Using "practical" time unit $\tau$, we can define "practical" distance unit $x$ as

$$
x=c \tau,
$$

where $c$ is the speed of light.

By this definition, we connect space metric to the time metric. This connection is formal and does not established physical alikeness of space and time. A principal difference is that space is reversible while time is not. Formally, we can proceed even farther and convert Euclidian space-time into Minkowski's spacetime using Wick's rotation, $t \rightarrow-i t$. In this case, definition (5.2) should be replaced with this one

$$
x=-i c \tau .
$$

Formally, Wick's rotation would transform dissipative processes, described by the real time exponents, into periodical processes, described by imaginary exponents. i.e. the self-organized vacuum would be transformed into a conservation obeying medium, something resembling vacuum employed by the relativity theory or standard model of particle interactions.

With or without Wick's rotation, "practical" time gauged by $\tau$ resembles dilated time in general relativity. Drawing a parallel between the proposed model and general relativity, one can find that the flat space-time happens at superattractors, and the black holes emerge at bifurcations. Remarkably, the black hole emergence is accompanied by phase transitions from one type of fundamental interactions to another, starting from emergence of cellular structure (electromagnetic interactions) through emergence of weak, and strong fields. The initial state is lack of electromagnetic interactions and we call it "dark substance". It locates in Z0 or "the dark zone". 


\subsection{The Dark Zone}

In this section we briefly explore interval $-1<B<-0.5$, which is located at the left of $Z 1 L$ in the bifurcation diagram. This is vacuum state that gives birth to all fundamental interactions. We call it the dark zone. Another reason to give this name is that in $Z 0$, amplification parameter $A<1$, and vacuum dust selfrepelling is not balanced by its self-attraction. The vacuum dust progressively disperses when we approach the superattractor $(B \rightarrow-1)$, i.e. behave like under action of the dark energy.

The dark zone has some similarities with the other zones, but it also has a lot of differences. $\chi(t)$-trajectories converge to a fixed point (Figure 46(left)), and this is the only fixed point, $\chi_{\infty}=-1$. The trajectories belonging to different $B$ values always entangle with each other. They exponentially converge toward the only fixed point (Figure 46(right)). However, their slopes $\lambda$ are different for different $B$ values. Formally, we can define energy $\mathcal{E}$ relaxation time $\tau$, and delayed "practical time scale" using the same definitions as we used for the other zones.
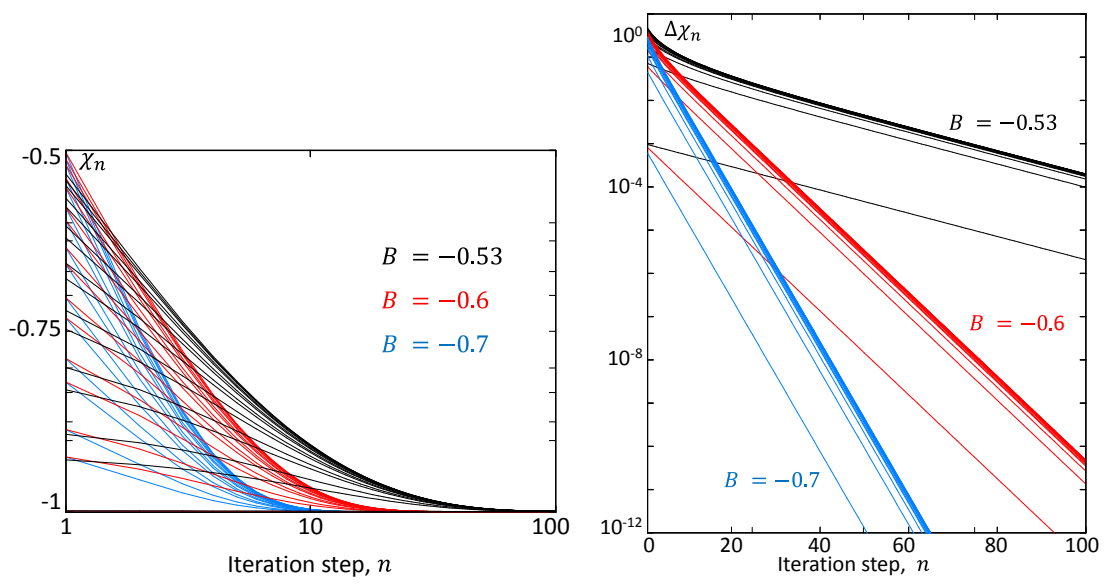

Figure 46. Examples of evolution of radial flows $\chi$ (left). Examples of evolution of uncertainty intervals $\Delta \chi$ in zone $Z 0$ (right).

Using (2.19) we can define parameter $\beta$ to find out that functions $\mathcal{E}(\beta)$ and $\tau(\beta)$ are practically the same as in the other zones (Figure 47).
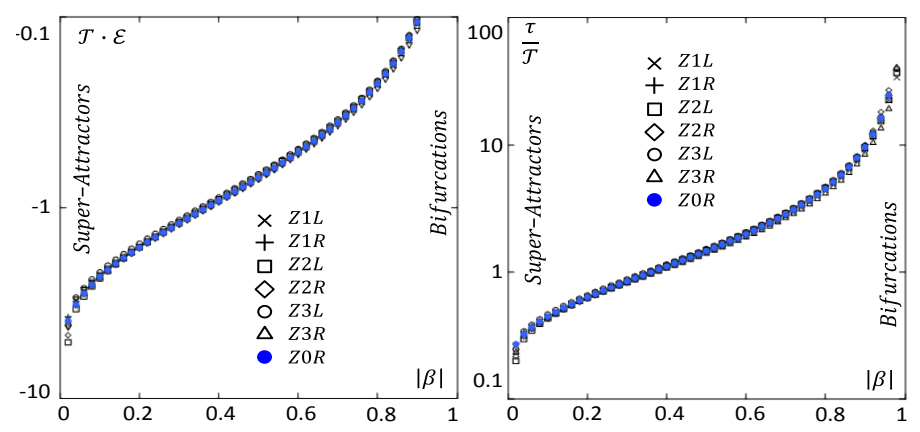

Figure 47. $\mathrm{n}(|\beta|)$ and $\tau(|\beta|)$ in all semi-zones. Dark zone data are shown by blue circles. 
The dark zone possess a strange stability. From the state-space point of view, this is a zone of asymptotic stability. Indeed, formally all trajectories asymptotically converge toward the unique fixed point (which is also the superattractor). From the real physical space point of view, the vacuum dust unboundly disperse across the space. The closer the dust locates to the fixed point the stronger is the dispersion rate in space. In the dark zone, vacuum dust behaves like a chaotic system. The exponential expansion of the dust in space also resembles the Hubble law.

\section{Concluding Remarks}

In contrast to the existing tradition, we introduced a new dynamical model without any reference to Hamiltonian, Lagrangian, or variational principle. In the proposed model, the role of those is delegated to the iteration functions $\mathcal{L} j$, which comprise all what we need to describe the vacuum cells evolution.

Curiously, in each zone, $\mathcal{L} j$ can be written in a form resembling Lagrangian as difference between two functions, $\mathcal{P} j$ and $\mathcal{K} j$ (see (6.1)). The former is independent of variable $\chi$, represents external forces (through control parameter $B$ ) and can be associated with the system potential energy. The other explicitly depends on $\chi^{2}$, reflects the system reaction (through variable $\chi$ ), and can be associated with kinetic energy.

$$
\mathcal{L} j=\mathcal{P} j-\mathcal{K} j
$$

where

$$
\begin{aligned}
\mathcal{P} 1= & B \\
\mathcal{K} 1= & (1+B) \chi^{2} \\
\mathcal{P} 2= & -B\left(B^{2}+B-1\right) \\
\mathcal{K} 2= & -2 B(B+1)^{2} \chi^{2}+(B+1)^{3} \chi^{4} \\
\mathcal{P} 3= & -B\left(B^{2}+B-1\right)\left(B^{12}+6 B^{11}+12 B^{10}+5 B^{9}-12 B^{8}-12 B^{7}+B^{6}-4 B^{4}+B^{3}+2 B^{2}+1\right) \\
\mathcal{K} 3= & -8 B^{3}(B+1)^{4}\left(B^{8}+4 B^{7}+3 B^{6}-5 B^{5}-5 B^{4}+3 B^{3}+B^{2}-2 B+1\right) \chi^{2} \\
& +4 B^{2}(B+1)^{5}\left(7 B^{8}+28 B^{7}+27 B^{6}-17 B^{5}-29 B^{4}+3 B^{3}+5 B^{2}-4 B+1\right) \chi^{4} \\
& -8 B^{2}(B+1)^{7}\left(7 B^{6}+21 B^{5}+11 B^{4}-13 B^{3}-7 B^{2}+3 B-1\right) \chi^{6} \\
& +2 B(B+1)^{8}\left(35 B^{6}+105 B^{5}+75 B^{4}-25 B^{3}-27 B^{2}+3 B-1\right) \chi^{8} \\
& -8 B^{2}(B+1)^{11}\left(7 B^{2}+7 B-3\right) \chi^{10}+4 B(B+1)^{12}\left(7 B^{2}+7 B-1\right) \chi^{12} \\
& -8 B(B+1)^{14} \chi^{14}+(B+1)^{15} \chi^{16}
\end{aligned}
$$

Newtonian, Lagrangian, Hamiltonian equations of motions in classical mechanics, Feynman's pass integrals in quantum mechanics, Einstein-Hilbert general relativity equations can be "derived" with use of variational principles. The final results allow us to determine classical trajectories or most probable quantum states. Remarkably, we can formulate a "variational principle" for the vacuum cell dynamics. We have seen that the most probable locations for the vacuum cells in the state-space are super attractors. They always locate at the local 
extrema of the corresponding iteration function that is graphically illustrated in Figure 48. The pictures show graphical solutions of equations for super attractors $(B=S j)$ in each of the three zones:

$$
\left\{\begin{array}{l}
\mathcal{L} j\left(\chi_{\infty}^{2}, S j\right)= \pm \chi_{\infty} \\
+ \text { for cells } \\
\text {-for anticells }
\end{array}\right.
$$

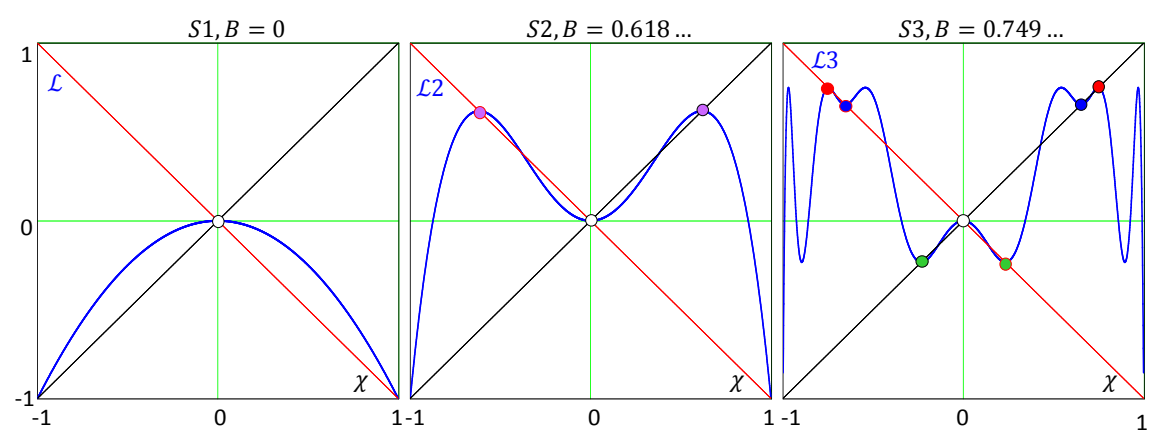

Figure 48. Equation (6.2) graphical solutions for $B=S j$.

Left parts of equations, $\mathcal{L} j$, are represented by blue curves, and right parts, $\pm \chi_{\infty}$, are represented by black and red diagonals. All stable solutions can be found at $\mathcal{L} j$ local extrema

$$
\frac{\partial \mathcal{L} j}{\partial \chi}=0
$$

They are shown by small circles, white-for neutral states, and colored-for charged states.

Another conventional way to find location of stable states is to determine minimum of potential energy. Applying to the cell dynamics, we can use "potential functions" $\mathcal{P} j$ defined in (6.1) to find the locations of super attractors. However, instead of function minima, we are looking for their roots (see Figure 49).

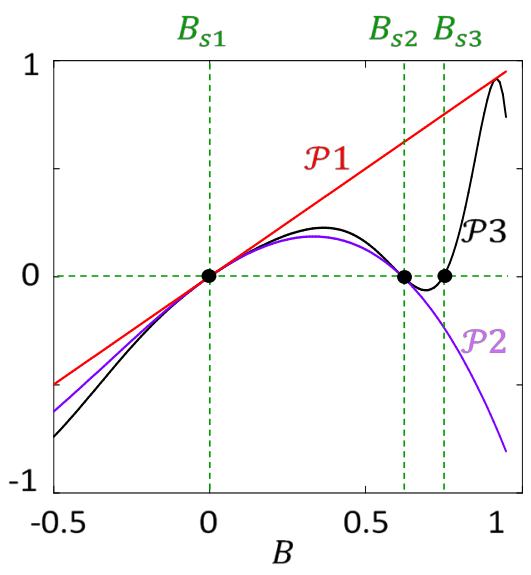

Figure 49. Finding superattractors $B_{s j}$ as roots of potential functions, $\mathcal{P} j=0$. 
However, square of the potential functions form "potential wells" with mini$\mathrm{ma}$ at the superattractors (Figure 50).

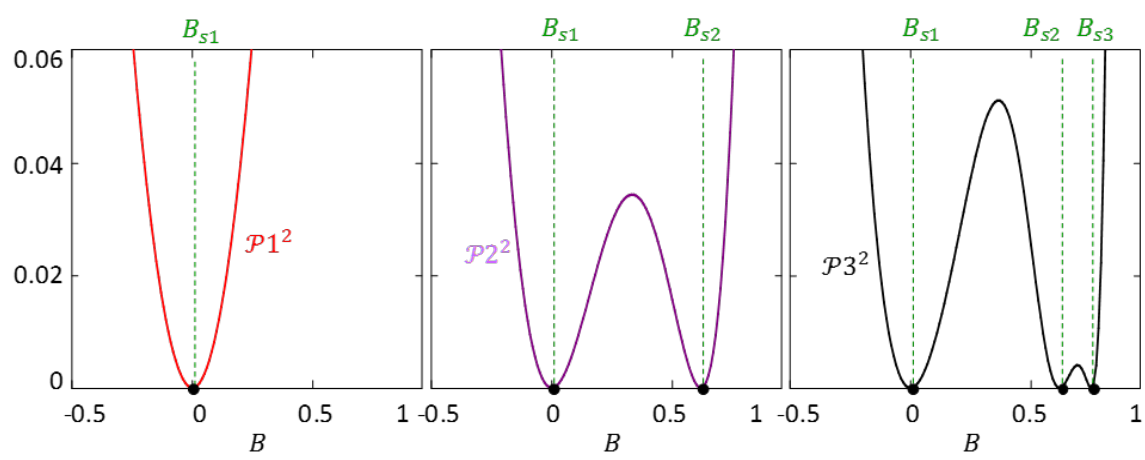

Figure 50. $\mathcal{P j}^{2}$ form potential wells with superattractor states at their bottoms.

When we are talking about particle interactions such as weak or strong nuclear interactions, do we really believe that Nature is that intelligent that she invented the Dirac or Yang-Mills equations? Can we consider the standard model, or super-gravity, or strings, which operate with highly developed math as fundamental laws that control the universe?

Let us for a second assume that the toy particles are real, that we learned about their properties including explicit form of equations (6.1) from fancy experiments without any clue that they can be obtained by using a simple iteration process. Would we call (6.1) the fundamental Nature equations? Perhaps.

But we do know that the complexity and symmetries of the toy particles stem from dull iterations of a primitive function. We have observed quantum behavior, complex structures, symmetries and physical constants emergent on premises of system openness, dissipation, randomness, and competition rather than according to some intelligent design, in a striking similarity to Darwin's evolution.

\section{Acknowledgements}

The paper is dedicated to the memory of my parents.

It would never be written if I did not meet my math teacher Isaak Rotfort and my university mentor Fernando Wilf. This project was born in connection with my daughter Julia's discovery of curiously strange correlations in chaotic networks that resembles remote particle entanglement [49]. The proposed model went through a number of iterations under the influences of numerous papers, books, and Internet sources. The most relevant literature is listed below. I owe thanks to my colleagues Vladimir Litvinov and Lev Sadovnik, and my children Julia and Alexander for stimulating discussions, comments, and suggestions.

\section{References}

[1] Hieb, M. (1995) Feigenbaum's Constant and the Sommerfeld Fine-Structure Constant. Unpublished.

[2] Goldfain, E. (2006) Chaos, Solitons \& Fractals, 30, 324-331. 
https://doi.org/10.1016/j.chaos.2006.01.117

[3] Manasson, V.A. (2006) Self-Interacting Electron as a Nonlinear Dynamical System. https://arxiv.org/abs/nlin/0609043 [nlin.CD]

[4] Manasson, V. A. (2008) Are Particles Self-Organized Systems? https://arxiv.org/abs/0803.3300 [physics.gen-ph]

[5] Feigenbaum, M.J. (1978) Journal of Statistical Physics, 19, 25-52. https://doi.org/10.1007/BF01020332

[6] Feigenbaum, M.J. (1979) Journal of Statistical Physics, 21, 669-706. https://doi.org/10.1007/BF01107909

[7] Coullet, P. and Tresser, C. (1978) Le Journal de Physique Colloques, 539C, 5-25; C.R. Acad. Sci. Paris, 287A, 577-580.

[8] Grossmann, S. and Thomae, S. (1977) Z. Naturforsh, 32a, 1353-1363.

[9] Collet, P. and Eckmann, J.-P. (1980) Iterated Maps on the Interval as Dynamical Systems. Birkhauser, Basel.

[10] Cvitanović, P. (1984) Universality in Chaos. Adam Hilger, Ltd., Bristol.

[11] Cvitanović, P. (1984) Acta Physica Polonica, A65, 203-239.

[12] Hilborn, R.C. (1994) Chaos and Nonlinear Dynamics: An Introduction for Scientists and Engineers. Oxford University Press, New York, Oxford.

[13] Strogatz, S.H. (1994) Nonlinear Dynamics and Chaos: With Applications to Physics, Biology, Chemistry, and Engineering. Perseus Books Publishing, LLC, Westview.

[14] Sfondrini, A. (2013) Introduction to Universality and Renormalization Group Techniques. Proceedings of Science Pos (Modave VIII), 005, Arxiv: 1210.2262v3 [Hep-Th] 25 Jun 2013.

[15] Griffiths, D.J. (1987) Introduction to Elementary Particles. John Willey \& Sons, Hoboken. https://doi.org/10.1002/9783527618460

[16] Physical Constants (2013) Particle Data Group Review. http://pdg.lbl.gov/2017/reviews/rpp2016-rev-phys-constants.pdf

[17] The CKM Quark-Mixing Matrix (2014) Particle Data Group Review. http://pdg.lbl.gov/2014/reviews/rpp2014-rev-ckm-matrix.pdf

[18] Benettin, G., Cercignani, C. Galgani, L. and Giorgilli, A. (1980) Lettere al NuovoCimento, 28, 1-4. https://doi.org/10.1007/BF02776065

[19] Greene, J.M., Mackay, R.S., Vivaldi, F. and Feigenbaum, M.J. (1981) Physica D. Nonlinear Phenomena, 3, 468-486. https://doi.org/10.1016/0167-2789(81)90034-8

[20] Bountis, T. (1981) Physica D: Nonlinear Phenomena, 3, 577-589. https://doi.org/10.1016/0167-2789(81)90041-5

[21] Widom, M. and Kadanoff, L. (1982) Physica D: Nonlinear Phenomena, 5, 287-292.

[22] Mackay, R.S. (1993) Renormalisation in Area-Preserving Maps. World Scientific, Singapore. https://doi.org/10.1142/2001

[23] Turing, A.M. (1952) Philosophical Transactions of the Royal Society of London. Series B, Biological Sciences, 237, 37-72. https://doi.org/10.1098/rstb.1952.0012

[24] Prigogine, I. and Nicolis, G. (1977) Self-Organization in Non-Equilibrium Systems. Wiley, New York.

[25] Haken, H. (1983) Advanced Synergetics: Instability Hierarchies of Self-Organizing Systems and Devices (Springer Series in Synergetics). Springer-Verlag, Berlin Heidelberg.

[26] Prigogine, I. and Stengers, I. (1984) Order Out of Chaos. Bantam, New York 
[27] Nicolis, G. and Prigogine, I. (1989) Exploring Complexity. W. H. Freeman and Co., New York.

[28] Wesfreid, J.E. (2006) Scientific Biography of Henri Bénard (1874-1939). In: Mutabazi, I., Wesfreid, J.E. and Guyon, E., Eds., Dynamics of Spatio-Temporal Cellular Structures, Springer, New York, 9-37. (See the List of Bénard's Papers and Description of His Experiments)

[29] Maurer, J. and Libchaber, A. (1979) Journal De Physique Lettres, 40, 419-423. https://doi.org/10.1051/jphyslet:019790040016041900

[30] Feigenbaum, M.J. (1979) Physics Letters A, 74, 375-378. https://doi.org/10.1016/0375-9601(79)90227-5

[31] Libchaber, A. and Maurer, J. (1980) Une Experience De Journal De Physique Colloques, 41, C3-51-C3-56.

[32] Libchaber, A., Laroche, C. and Fauve, S. (1982) Period Doubling Cascade in Mercury. A Quantitative Measurement. Journal De Physique Lettres, 43, 211-216. https://doi.org/10.1051/jphyslet:01982004307021100

[33] Yahata, H. (1983) Progress of Theoretical Physics, 69, 1802-1805. https://doi.org/10.1143/PTP.69.1802

[34] Winfree, A.T. (1987) When Time Breaks Down. Princeton University Press, Princeton.

[35] Walgraef, D. (1997) Spatio-Temporal Pattern Formation: With Examples from Physics, Chemistry, and Material Science. Springer-Verlag, New York. https://doi.org/10.1007/978-1-4612-1850-0

[36] Camazine, S., Deneubourg, J.-L., Franks, N.R., Sneyd, J., Theraulaz, G. and Bonabeau, E. (2001) Self-Organization in Biological Systems. Princeton University Press, Princeton and Oxford.

[37] Schöll, E. (2001) Nonlinear Spatio-Temporal Dynamics and Chaos in Semiconductors. Cambridge University Press, Cambridge. https://doi.org/10.1017/CBO9780511524615

[38] Solé, R.V. and Bascompte, J. (2006) Self-Organization in Complex Ecosystems. Princeton University Press, Princeton and Oxford.

[39] Adler, R. (1946) Proceedings of the IRE, 34, 351-357; Reprinted in Proceedings of the IEEE, 61, 1380-1385 (1973). https://doi.org/10.1109/PROC.1973.9292

[40] Strogatz, S.H. (2000) From Kuramoto to Crawford: Exploring the Onset of Synchronization in Populations of Coupled Oscillators. Physica D: Nonlinear Phenomena, 143, 1-20. https://doi.org/10.1016/s0167-2789(00)00094-4

[41] Pikovsky, A., Rosenblum, M. and Kurths, J. (2001) Synchronization. A Universal Concept in Nonlinear Sciences. Cambridge University Press, Cambridge. https://doi.org/10.1017/CBO9780511755743

[42] Balanov, A., Janson, N., Postnov, D. and Sosnovtseva, O. (2009) Synchronization: From Simple to Complex. Springer.

[43] Dörfler, F. and Bullo, F. (2014) Automatica, 50, 1539-1564. https://doi.org/10.1016/j.automatica.2014.04.012

[44] Penrose, R. (2004) The Road to Reality: A Complete Guide to the Laws of the Universe. Vintage Books, New York.

[45] Yamaguchi, Y. and Shimizu, H. (1984) Physica D: Nonlinear Phenomena, 11, $212-$ 226.

[46] Bar-Eli, K. (1985) Physica D: Nonlinear Phenomena, 14, 242-252. 
https://doi.org/10.1016/0167-2789(85)90182-4

[47] Mirollo, R.E. and Strogatz, S.H. (1990) Journal of Statistical Physics, 60, 245-262. https://doi.org/10.1007/BF01013676

[48] Koseska, A., Volkov, E. and Kurths, J. (2013) Physics Reports, 531, 173-199. https://doi.org/10.1016/j.physrep.2013.06.001

[49] Manasson, J. and Manasson, V.A. (2016) Strange Correlations between Remote Nodes in Networks Comprising Chaotic Links. https://arxiv.org/abs/1511.02772 [nlin.CD]

Submit or recommend next manuscript to SCIRP and we will provide best service for you:

Accepting pre-submission inquiries through Email, Facebook, LinkedIn, Twitter, etc. A wide selection of journals (inclusive of 9 subjects, more than 200 journals) Providing 24-hour high-quality service User-friendly online submission system Fair and swift peer-review system Efficient typesetting and proofreading procedure Display of the result of downloads and visits, as well as the number of cited articles Maximum dissemination of your research work

Submit your manuscript at: http://papersubmission.scirp.org/ Or contact jmp@scirp.org 